\title{
A Survey on Brain Biometrics
}

\author{
QIONG GUI, MARIA V. RUIZ-BLONDET, and SARAH LASZLO, Binghamton University,
}

State University of New York

ZHANPENG JIN, University at Buffalo, State University of New York

\begin{abstract}
Brainwaves, which reflect brain electrical activity and have been studied for a long time in the domain of cognitive neuroscience, have recently been proposed as a promising biometric approach due to their unique advantages of confidentiality, resistance to spoofing/circumvention, sensitivity to emotional and mental state, continuous nature, and cancelability. Recent research efforts have explored many possible ways of using brain biometrics and demonstrated that they are a promising candidate for more robust and secure personal identification and authentication. Although existing research on brain biometrics has obtained some intriguing insights, much work is still necessary to achieve a reliable ready-to-deploy brain biometric system. This article aims to provide a detailed survey of the current literature and outline the scientific work conducted on brain biometric systems. It provides an up-to-date review of state-of-the-art acquisition, collection, processing, and analysis of brainwave signals, publicly available databases, feature extraction and selection, and classifiers. Furthermore, it highlights some of the emerging open research problems for brain biometrics, including multimodality, security, permanence, and stability.
\end{abstract}

CCS Concepts: • Security and privacy $\rightarrow$ Biometrics; • Computing methodologies $\rightarrow$ Machine learning approaches; • Applied computing $\rightarrow$ Psychology;

Additional Key Words and Phrases: Biometric, brain, brainprints, identification, authentication

ACM Reference format:

Qiong Gui, Maria V. Ruiz-Blondet, Sarah Laszlo, and Zhanpeng Jin. 2019. A Survey on Brain Biometrics. ACM Comput. Surv. 51, 6, Article 112 (February 2019), 38 pages.

https://doi.org/10.1145/3230632

\section{INTRODUCTION}

Means of authentication and identification can be classified into three groups: something the user knows, something the user has, and something the user is [123]. Traditionally widely used methods in the categories of something the user knows and has, like passwords and ATM cards, have

This work was supported in part by the National Science Foundation (NSF) under the grants CNS-1422417 (Z. Jin \& S. Laszlo), CNS-1564046 (Z. Jin \& S. Laszlo), CNS-1840790 (Z. Jin), and BCS-1252975 (S. Laszlo), as well as in part by the Binghamton University Interdisciplinary Collaboration Grants (ICG) program and Health Sciences Transdisciplinary Area of Excellence (TAE) Seed Grant program.

Authors' addresses: Q. Gui, M. V. Ruiz-Blondet, and S. Laszlo, Binghamton University, State University of New York, 4400 Vestal Pkwy East, P. O. Box 6000, Binghamton, NY, 13902-6000, USA; emails: \{qgui1, mruizbl1\}@binghamton.edu, cogneuro@alum.mit.edu; Z. Jin, University at Buffalo, State University of New York, Department of Computer Science and Engineering, 338 Davis Hall, Box 602000, Buffalo, NY, 14260-2500, USA; email: zjin@buffalo.edu.

Permission to make digital or hard copies of all or part of this work for personal or classroom use is granted without fee provided that copies are not made or distributed for profit or commercial advantage and that copies bear this notice and the full citation on the first page. Copyrights for components of this work owned by others than ACM must be honored. Abstracting with credit is permitted. To copy otherwise, or republish, to post on servers or to redistribute to lists, requires prior specific permission and/or a fee. Request permissions from permissions@acm.org.

(C) 2019 Association for Computing Machinery.

0360-0300/2019/02-ART112 \$15.00

https://doi.org/10.1145/3230632 
demonstrated clear weaknesses. Tokens the user knows or has can be forgotten, lost, or stolen, leading to unsuccessful authentication or information leakage. Biometrics, which is a means that the user is, can overcome these drawbacks. Biometrics do not require any memorizing because they are innate physiological or behavioral parts of the individual. Biometrics cannot be lost or stolen for the same reason.

Currently, conventional biometrics, such as fingerprint, face, iris, voice, and DNA, have been extensively studied in literature and widely adopted in real-life scenarios. However, these biometrics each possess their weaknesses [59]. For example, DNA can be easily stolen from any surface a target has touched. Fingerprints can be faked through plastic molds, latex milk, and wood glue [99]; by a 2D picture of a fingerprint made by a normal inkjet printer [21]; and even through highresolution photography [23]. Fingerprints, face, and iris are all noncancelable. This means that if they are stolen, they cannot be replaced: a user cannot grow a new finger or eye or change the shape of her face volitionally. A biometric more secure than any of these would therefore meet two criteria: it would be more difficult to steal, and it would be cancelable. Brain electrical activity measured at the scalp (throught an electroencephalogram or EEG) may meet these criteria.

Since EEG was first developed in 1929, many studies have shown that brain activity can be influenced by many genetic and nongenetic factors and have demonstrated high individuality of EEG and Event-Related Potentials (ERPs) (please see Section 4.1 for differences between EEG and ERP) among different people [170]. This finding indicates that the brainwave signal could be unique for each individual and thus has the potential to be a biometric [19]. The EEG has several unique advantages compared with other conventional biometrics. First, in order to produce EEG, a person must be alive-the lack of an EEG is the primary clinical indicator of brain death [175, 176]. This is not the case for DNA, fingerprint, and face, all of which can be maintained even after people die, or the iris, which, though eventually changing after death, is still valid for recognition for a few hours post mortem $[149,169]$. In this respect, brain biometrics also protect the user, as the user has to be alive and conscious state to produce valid EEG data. Second, brain electrical activity is measured as a voltage which falls off dramatically with distance from the brain. Therefore, the electrodes to record brain activity must be placed either on the scalp, using wet or dry noninvasive sensors [25, 26], or close to the scalp, using remote measurement of electric fields [48]. Current sensors are large and invasive enough that it would be impossible to collect the EEG without a person's knowledge [96, 142]. Thus brain biometrics meet the criteria of being "more difficult to steal." Third, recent research suggests that brain biometrics may be cancelable [142] because they can be elicited by numerous distinct brain systems. For example, when the stored brain biometric identifier of an authorized user is lost, stolen, or disclosed, a different brain biometric identifier can be generated from a distinct form of brain activity and response (e.g., ERP evoked by a distinct set of visual stimuli) and thus can be used to replace the compromised and corrupted one. Fourth, the EEG is very sensitive to stress [22]. This is another factor that may protect the user of a brain biometric system: Any attempt at coercion that would place stress on the user would invalidate the brain biometric. Finally, high-accuracy identification can be achieved with nonvolitional features of the EEG, meaning that brain biometrics are not disclosable: A user cannot reveal a biometric feature that they are not aware of $[142,146]$. Given the unique and superior advantages described above, brain biometrics holds the potential to be used in the scenarios demanding a very high security level, such as in defense and financial agencies.

Despite the many advantages of brain biometrics, they are still not widely adopted because substantial research still must be done, and reviewing the areas of necessary research is one focus of this review. There are seven factors-universality, uniqueness, permanence, collectability, performance, acceptability, and circumvention [58, 59]-that evaluate how reasonable a biometric is as an authentication or identification method. Many prior research efforts have demonstrated 
Table 1. Comparison Between Brain Biometrics and Some Popular Biometrics

\begin{tabular}{cccccccc}
\hline Biometric Identifier & Universality & Uniqueness & Permanence & Collectability & Performance & Acceptability & Circumvention \\
\hline DNA & H & H & H & L & H & L & L \\
Face & H & L & M & H & L & H & H \\
Fingerprint & M & H & H & M & H & M & M \\
Iris & H & H & H & M & H & L & L \\
Brainwave & H & H & H & M & H & M & H \\
\hline
\end{tabular}

satisfactory uniqueness, permanence, and universality for brain biometrics. Recent research has also shown that brain biometric is resistant to spoofing and circumvention from the perspectives of signal acquisition and synthesis [45]. However, more research is needed to improve the collectability, performance, and acceptability of brain biometrics [19]. Table 1 compares the brain biometrics against some popular biometrics in terms of the seven factors defined in Jain et al. [59]. However, there are very few existing surveys that comprehensively review the scientific rationale of brain biometrics and the various protocols for eliciting, acquiring, processing, and analyzing brain activity signals. Some existing surveys, such as those by Khalifa et al. [70], Pozo-Banos et al. [134], and Campisi and La Rocca [19], discussed feature extraction, protocol design, and classifiers for brain biometric systems from multiple perspectives. As brain biometrics are becoming more feasible, it is important to take stock of what is and is not known about them. The goal of this survey is to expand on previous surveys by:

(1) reviewing state-of-the-art brain biometric acquisition devices and methods,

(2) reviewing publicly available databases for brain biometric studies,

(3) presenting preprocessing and postprocessing methods useful for biometric applications,

(4) describing effective methods for channel/feature selection and dimensionality reduction that reduce the complexity of the authentication problem,

(5) reviewing existing statistical and machine learning classifiers for brain biometrics,

(6) exploring multimodal biometric systems that include brain biometrics, and

(7) reviewing what is known about the security, permanence, and stability of brain biometrics.

In this article, we survey the recent literature (primarily between 2007 and 2017) and outline the scientific work conducted on brain biometric systems. This includes review of the acquisition, processing, and analysis of the EEG. This article is organized as follows: We first give a brief introduction to brain biometric systems (Section 2). Then we describe each part of the typical system in detail, including EEG acquisition devices and methods (Section 3), collection protocols and publicly available databases (Section 4), preprocessing/postprocessing techniques (Section 5), frequently used features (Section 6), channel/feature selection and dimensionality reduction selection (Section 7), and classifiers (Section 8). In conclusion, we highlight some of the open research areas in the field, including multimodal biometrics which have EEG as one of the biometric traits, and work investigating the security, permanence, and stability of brain-based biometrics (Section 9).

\section{BRAIN BIOMETRIC RECOGNITION SYSTEM}

Biometric authentication is the security process that verifies an individual's identity through unique physiological or behavioral characteristics. This process basically compares a biometric data capture against stored, confirmed authentic data in the database. Biometric identification, in contrast, represents the security process of identifying and labeling the identity of an individual by 


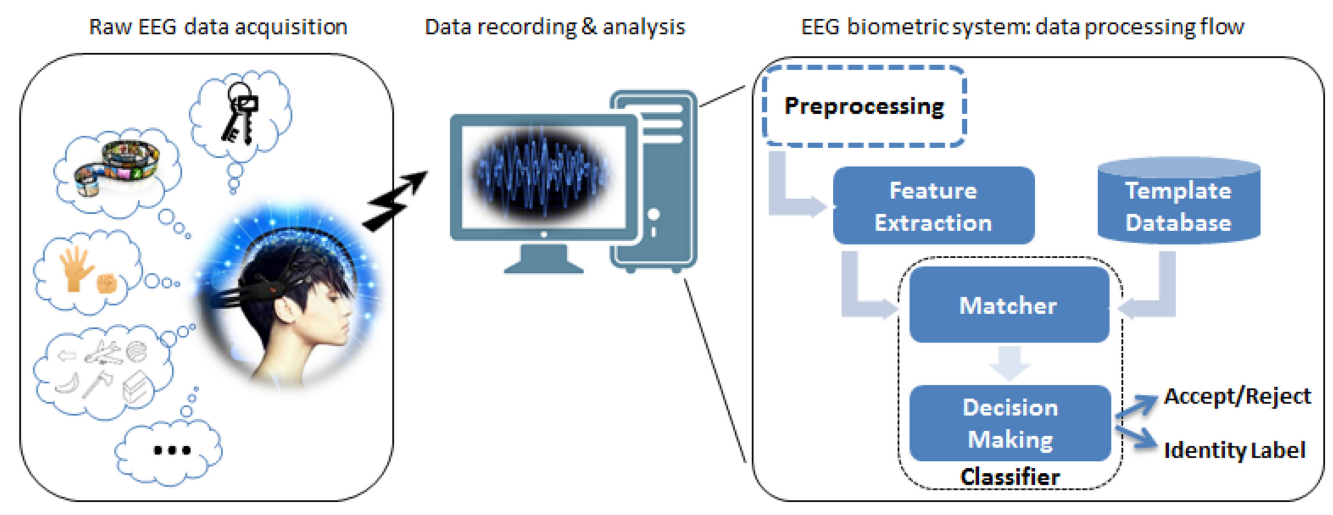

Fig. 1. General structure of brain biometric identification and authentication.

searching against a biometric database to find the distinctive biometric characteristics attributable to a single individual [57].

Brain biometric systems typically contain two parts, both for authentication [71] and identification applications [30]. These are (i) the data acquisition part and (ii) the decision part. Figure 1 shows a schematic architecture of brain biometric systems. In the data acquisition stage, EEG sensors capture brain electrical activity, while the user engages with some protocols, such as resting, pass-thought, visual stimulation, or imagery. Data are transferred for digitization and decision- making; this can occur wirelessly or with wired sensors. Once the data are digitized, the decision-making stage begins. Because the collected EEG data are usually contaminated with different kinds of noise and EEG data have a relatively low Signal-to-Noise Ratio (SNR), the first step in decision-making often involves signal preprocessing to enhance signal quality. Then various computational features are extracted. More often, the channel/feature selection and dimensionality reduction methods are adopted to reduce the dimensionality and computational complexity of the EEG data, which are typically large-dimensional [96]. When the feature set has been determined and confirmed, the biometric computations are performed. These may be simple statistical analyses or more complex machine learning approaches (e.g., Neural Network [NN], Support Vector Machine [SVM]). When the system is performing authentication, its output will be a binary acceptance/rejection. When the system is performing identification, its output will be the identity label of the user. More often in recent literature, nonlinear classifiers, like NN and SVM, can complete both the matching score calculation and decision-making in one classifier. Thus, they combine the matcher module and decision-making module into one module. In the following sections, we will review each of the steps just described. This includes review of EEG devices, EEG acquisition protocols, preprocessing, feature extraction, channel/feature selection and dimensionality reduction selection, and classifiers.

\section{EEG DEVICES AND COLLECTABILITY}

Collectability of the brainwaves specifies and requires that they should be practically and quantitatively measurable [19]. The brain's electrical currents were first observed from the exposed brains of rabbits and monkeys by an English physician named Richard Caton in 1875. In 1924, Hans Berger, a German neurologist, first recorded human brain electrical activity. The technology necessary for acquiring the EEG has thus been available for nearly a hundred years, although, of course, it has undergone substantial refinement since its initial discovery [12, 56, 161, 165]. 
Table 2. Commercially Available EEG Devices in Brain Biometric Studies

\begin{tabular}{|c|c|c|c|c|c|c|}
\hline $\begin{array}{l}\text { Device } \\
\text { (Company) }\end{array}$ & Channels & Sampling Rate & Bandwidth & Connectivity & Battery Life & Papers \\
\hline $\begin{array}{l}\text { ActiveTwo } \\
\text { (Biosemi) }\end{array}$ & 256 & $2 / 4 / 8 / 16 \mathrm{kHz}$ & $\begin{array}{l}\text { DC up to } 3200 \\
\mathrm{~Hz}\end{array}$ & Cable & $\begin{array}{l}5 \text { hrs to } 1 \text { week } \\
\text { (battery box) }\end{array}$ & {$[35,100,118]$} \\
\hline $\begin{array}{l}\text { BrainAmp } \\
\text { (Brain Vision) }\end{array}$ & up to 256 & $5 \mathrm{kHz}$ & $0-1,000 \mathrm{~Hz}$ & Cable & $\begin{array}{l}\text { up to } 30 \mathrm{hrs} \\
\text { (powerpack) }\end{array}$ & $\begin{array}{l}{[20,24,79,80,} \\
142]\end{array}$ \\
\hline $\begin{array}{l}\text { ENOBIO } \\
\text { (Neuroelectrics) }\end{array}$ & $8 / 20 / 32$ & $500 \mathrm{~Hz}$ & $0-125 \mathrm{~Hz}$ & Bluetooth & $16 / 15 / 14 \mathrm{hrs}$ & {$[66,138]$} \\
\hline $\begin{array}{l}\text { EPOC } \\
\text { (Emotiv) }\end{array}$ & 14 & $128 \mathrm{~Hz} / 256 \mathrm{~Hz}$ & $0.2-43 \mathrm{~Hz}$ & Bluetooth & up to $12 \mathrm{hrs}$ & $\begin{array}{l}{[7,49,61,67,73,} \\
75,76,109,168, \\
186]\end{array}$ \\
\hline $\begin{array}{l}\text { Galileo BE Light } \\
\text { (EB Neuro) }\end{array}$ & 37 & up to $32 \mathrm{kHz}$ & N/A & Cable, Wi-Fi & N/A & $\begin{array}{l}{[32-34,92,97,} \\
130]\end{array}$ \\
\hline $\begin{array}{l}\text { g.MOBIlab+ } \\
\text { (G.Tech Medical } \\
\text { Engineering } \\
\text { GmbH) }\end{array}$ & 8 & $256 \mathrm{~Hz}$ & $0.5-100 \mathrm{~Hz}$ & Bluetooth & $25-100 \mathrm{hrs}$ & [1] \\
\hline $\begin{array}{l}\text { g.USBamp } \\
\text { (G.Tech Medical } \\
\text { Engineering } \\
\text { GmbH) }\end{array}$ & 16 & up to $38.4 \mathrm{kHz}$ & $0-2,400 \mathrm{~Hz}$ & Cable & N/A & $\begin{array}{l}{[24,36,124,177,} \\
178]\end{array}$ \\
\hline $\begin{array}{l}\text { MindWave } \\
\text { (Neurosky) }\end{array}$ & 1 & $512 \mathrm{~Hz}$ & $3-100 \mathrm{~Hz}$ & Bluetooth & $8 \mathrm{hrs}$ & {$[2,28,30,62]$} \\
\hline $\begin{array}{l}\text { SynAmps 2/RT } \\
\text { (Compumedics/ } \\
\text { NeuroScan) }\end{array}$ & $64 / 128 / 256$ & up to $20,000 \mathrm{~Hz}$ & $\begin{array}{l}\text { DC up to } 3,500 \\
\mathrm{~Hz}\end{array}$ & Cable & N/A & {$[50,51,89,182]$} \\
\hline
\end{tabular}

To capture the EEG, an EEG device must include sensors that can make conductive contact with the scalp, a bioamplifier that enables online filtering and common mode rejection, an A/D converter, and a PC to store the digitized data. Table 2 lists several commercially available EEG devices used in the studies of brain biometrics. A typical complete system for EEG recording, such as the ActiveTwo system by BioSemi (Amsterdam, Netherlands) ${ }^{1}$, is usually a wired system that can accommodate as many as 256 channels. In the ActiveTwo system, sensors are placed in an electrocap and a conductive gel is applied to make contact between the sensors and the scalp. The ActiveTwo system uses active amplification, which means that signals are preamplifed on the sensor before being passed to the bioamplifier. This reduces the impact of electrical noise that is induced in the wires connecting the sensors to the amplifier. The ActiveTwo system enables highdensity recordings from as many as 256 sensors. This is an advantage because it provides more spatial coverage of the scalp and, consequently, a richer dataset.

A disadvantage of high-density recording, however, is that the preparation process is long. A conductive connection with the scalp must be made individually for each sensor. This is timeconsuming. High-density recordings are also more expensive, as, obviously, each sensor adds to the cost of the unit. Research-grade systems, in general, are too expensive for consumer use, which is another disadvantage. The main cost is the bioamplifier.

Recent advances in large-scale integrated circuits and wireless communication have resulted in the development of cheaper, more consumer-friendly EEG devices. Examples of these are the ENOBIO developed by Neuroelectrics (Barcelona, Spain) ${ }^{2}$, the EPOC/EPOC+ wearable

\footnotetext{
${ }^{1}$ http://www.biosemi.com/.

${ }^{2}$ http://www.neuroelectrics.com/.
} 
neuroheadset developed by Emotiv Systems, Inc. (San Francisco, USA) ${ }^{3}$, and the MindWave wearable headset developed by NeuroSky, Inc. (San Jose, USA) ${ }^{4}$. Consumer devices, however, typically do not allow recordings that are as high density as research-grade devices. And the cheaper sensors and bioamplifiers typically result in substantially reduced signal quality. There is thus a tradeoff between consumer- and research-grade systems. Consumer systems are cheaper and more user friendly, but produce poorer data. Research-grade systems produce high-density, high-quality data but are more expensive and less user friendly. These factors contribute to the "collectability" of brain biometrics. However, compared with extensively deployed fingerprint sensors or face recognition cameras, EEG devices still demand significant improvement in affordability, ease-of-use, and portability.

\section{DATA COLLECTION PROTOCOLS}

\subsection{Protocols}

EEG can be collected while a participant engages in any type of cognitive task or while the user simply rests with their eyes open (RO) or eyes closed (RC). The task the participant is engaging in will impact the nature of the data recorded, so it is important to define the task, or protocol, used in any biometric system. Table 3 summarizes the protocols used in a selection of published brain biometric approaches, and Table 4 lists some publicly available databases, including their protocols. Generally, protocols can be categorized into two types: resting states and cognitive tasks.

Resting-state EEG is acquired when the participant is simply at rest during data collection, not performing any particular task. Resting-state protocols are therefore simple and can be collected continuously. For this reason, resting-state protocols are common in brain biometrics $[1,20,30$, 78-81, 95-97, 157].

Cognitive protocols are more complex. In cognitive protocols, the participant engages in some specific tasks while the EEG is acquired. These tasks are many and varied in the literature and may involve specialized procedures. One type is mental tasks. These include pass-thoughts $[28,62]$ and mental imagery, such as mental rotation or imagining body movements [4, 7, 100, 112, 117]. In pass-thought and mental imagery protocols, the EEG is acquired while participants perform some internal, volitional tasks (e.g., imagining opening and closing a fist). It is also possible to acquire the EEG while participants are externally stimulated and capture the participants' nonvolitional response. The form of stimulation can vary, but the most common format is the presentation of human faces. Faces are used for multiple reasons. First, a person's brain response to his or her own face is different from his or her response to faces of others, and this information can be individuating $[105,177,178,182]$. Second, individuals differ in how attractive they find the faces of others, and this information can also be individuating [142].

Biometric systems using a cognitive protocol typically rely not on the raw EEG time series, but instead on the time-locked ERPs. ERPs refer to the scalp-recorded voltage fluctuations locked in time to an externally defined event. ERPs are formed when segments of the EEG time-locked to several instances of a stimulating event are averaged. For example, an ERP is formed when several EEG segments all locked to the presentation of a different face are averaged together. Other types of stimulation-evoked brain responses include Visual Evoked Potentials (VEP), Auditory Evoked Potentials (AEP), and Somatosensory Evoked Potentials (SSEP). Some prior brain biometric studies have also used the VEP protocol. Because VEPs are limited to the brain's visual cortex activities, they require very careful experimental design to lower the impact of other brain activities, such as semantic or auditory input. This is difficult to achieve; here, we use the concept of a wider scope

\footnotetext{
${ }^{3}$ http://emotiv.com/.

${ }^{4}$ http://neurosky.com/.
} 
Table 3. Typical EEG Collection Protocols

\begin{tabular}{|c|c|c|c|c|c|}
\hline Papers & Sbjs* & Chs* & Protocols & $\begin{array}{l}\text { Sampling } \\
\text { Rates }\end{array}$ & Sessions \\
\hline$[6,43,146]$ & 45 & 6 & acronym & $500 \mathrm{~Hz}$ & $\begin{array}{l}1 \text { ( } 30 \text { sbjs }) \\
2 \text { ( } 15 \text { sbjs, } 5-40 \text { days }) \\
3 \text { ( } 9 \text { sbjs, } 134-188 \text { days })\end{array}$ \\
\hline$[8,89]$ & 20 & 64 & $\begin{array}{l}\text { Revised Snodgrass and } \\
\text { Vanderwart object pictorial set }\end{array}$ & $1000 \mathrm{~Hz}$ & 1 \\
\hline$[20,78,80]$ & 48 & 56 & $\mathrm{RC}$ & $200 \mathrm{~Hz}$ & 1 \\
\hline$[28,62]$ & $15+3 I^{*}$ & 1 & $\begin{array}{l}\text { breathing task } \\
\text { simulated finger movement } \\
\text { sports task } \\
\text { song/passage recitation task } \\
\text { eye and audio tone task } \\
\text { object counting task } \\
\text { passthought task }\end{array}$ & $512 \mathrm{~Hz}$ & 2 (separate days) \\
\hline$[32-34]$ & 50 & 19 & $\begin{array}{l}\text { geometric shapes } \\
\text { letters \& digits }\end{array}$ & $256 \mathrm{~Hz}$ & $\begin{array}{l}1 \\
2 \text { (1 week) } \\
3 \text { (25-49 days) }\end{array}$ \\
\hline$[42,44]$ & 37 & 4 & $\begin{array}{l}\text { words, pseudowords, acronyms, } \\
\text { illegal strings }\end{array}$ & $500 \mathrm{~Hz}$ & 1 \\
\hline $\begin{array}{l}{[46,142,} \\
143]\end{array}$ & 50 & 30 & $\begin{array}{l}\text { sine gratings } \\
\text { low frequency words } \\
\text { black and white food } \\
\text { black and white celebrity faces } \\
\text { color version of black \& white images } \\
\text { color food }\end{array}$ & $500 \mathrm{~Hz}$ & $\begin{array}{l}1 \\
2 \text { (20 sbjs, } 48-516 \text { days })\end{array}$ \\
\hline$[50,51]$ & 7 & 19 & blocking virtual target balls & $1000 \mathrm{~Hz}$ & 1 \\
\hline [79] & 9 & 54 & $\mathrm{RC}, \mathrm{RO}$ & $200 \mathrm{~Hz}$ & 2 (1-3 weeks) \\
\hline [92] & 45 & 19 & $\begin{array}{l}\mathrm{RC}, \mathrm{RO}, \\
\text { mathematical computation, } \\
\text { speech imagery }\end{array}$ & $256 \mathrm{~Hz}$ & $\begin{array}{l}1 \\
2 \text { (about } 1 \text { week) } \\
3 \text { (about } 1 \text { month) } \\
4 \text { (about } 7 \text { months) } \\
5 \text { (about } 16 \text { months) } \\
6 \text { (30 sbjs, about } 36 \\
\text { months) }\end{array}$ \\
\hline [95-97] & 60 & 19 & $\mathrm{RC}, \mathrm{RO}$ & $256 \mathrm{~Hz}$ & $\begin{array}{l}1 \\
2 \text { (50 sbjs, about } 1 \text { week) } \\
3 \text { (50 sbjs, about } 34 \text { days) }\end{array}$ \\
\hline$[108,110]$ & 30 & 1 & driving scenes in virtual environment & $128 \mathrm{~Hz}$ & 10 (twice a day, 5 days) \\
\hline$[113,128]$ & 40 & 23 & free & $167 \mathrm{~Hz}$ & 1 \\
\hline$[157-160]$ & 40 & 1 & RC (water/coffee) & $200 \mathrm{~Hz}$ & $\begin{array}{l}6 \text { (water) } \\
6 \text { (coffee) }\end{array}$ \\
\hline$[177,178]$ & $15+30 I$ & 16 & self-face, non-self-face & $2400 \mathrm{~Hz}$ & 2 (30 days) \\
\hline
\end{tabular}

*Abbreviations used in all following tables: Sbjs - Subjects, Chs - Channels, I - intruders/imposters.

of ERP to include brain responses to not only visual stimuli, but also sensory, cognitive, motor, or other events.

ERPs have two clear advantages over the EEG for biometric use. First, the EEG is relatively unspecific: That is, when EEG is collected outside of a cognitive task, there is no way to know what the participant is thinking about or what state their brain is in. ERPs, in contrast, represent very specifically the brain's response to the stimulation that they are time-locked to. In the case of facial stimulation, for example, a face ERP represents precisely and only the brain's response to 
Table 4. Representative Publicly Available EEG Databases

\begin{tabular}{|c|c|c|c|c|c|c|}
\hline Databases & Sbjs & Chs & Protocols & $\begin{array}{l}\text { Sampling } \\
\text { Rate }\end{array}$ & Sessions & Papers \\
\hline Alcoholism & 122 & 64 & $\begin{array}{l}\text { Snodgrass \& Vanderwart } \\
\text { picture set }\end{array}$ & $256 \mathrm{~Hz}$ & 1 & $\begin{array}{l}{[55,60,87,113,} \\
181,188]\end{array}$ \\
\hline ATR & 51 & 64 & RO, left \& right attention & $256 \mathrm{~Hz}$ & 9 & {$[114]$} \\
\hline DEAP & 32 & $\begin{array}{l}\text { 32, GSR, BVP, } \\
\text { respiration, } \\
\text { skin temperature, } \\
\text { ECG, EMG, EOG }\end{array}$ & music video & $160 \mathrm{~Hz}$ & 1 & {$[86,103,129]$} \\
\hline EEGMMIDB & 109 & 64 & $\begin{array}{l}\text { RO, RC } \\
\text { open/close fists/feet } \\
\text { imagery opening/closing } \\
\text { fists/feet }\end{array}$ & $160 \mathrm{~Hz}$ & 1 & $\begin{array}{l}{[4,10,29,40,} \\
64,81,140,152, \\
166,167,179, \\
186]\end{array}$ \\
\hline Graz IIIa & 3 & 60 & $\begin{array}{l}\text { imagery left/right hand, } \\
\text { feet or tongue movements }\end{array}$ & $250 \mathrm{~Hz}$ & 1 & {$[113]$} \\
\hline Graz A & 9 & $22, \mathrm{EOG}$ & $\begin{array}{l}\text { imagery left/right hand, } \\
\text { feet or tongue movements }\end{array}$ & $250 \mathrm{~Hz}$ & 2 & $\begin{array}{l}{[74,111,113,} \\
127]\end{array}$ \\
\hline Graz B & 9 & $3, \mathrm{EOG}$ & $\begin{array}{l}\text { motor imagery left/right } \\
\text { hand movement }\end{array}$ & $250 \mathrm{~Hz}$ & 5 & {$[111-113,127]$} \\
\hline Keirn \& Aunon & 7 & 6, EOG & $\begin{array}{l}\text { RO, RC } \\
\text { Complex problem solving } \\
\text { 3-D geometric figure } \\
\text { rotation } \\
\text { Mental letter composing } \\
\text { Visual counting }\end{array}$ & $250 \mathrm{~Hz}$ & 1 & {$[117,139]$} \\
\hline $\begin{array}{l}\text { Two-class MI } \\
\text { BNCI Horizon } \\
2020\end{array}$ & 14 & 7 & $\begin{array}{l}\text { motor imagery of } \\
\text { right hand \& feet }\end{array}$ & $512 \mathrm{~Hz}$ & 1 & [74] \\
\hline
\end{tabular}

the face it has just been been presented. This is advantageous for biometric use because the brains of users of ERP protocols can be directed into states that are likely to be individuating (e.g., those corresponding to the recognition of their own face). The second advantage of ERPs over EEG for biometric use regards SNR properties. As ERPs are an averaged signal, their SNR is better than that of the EEG, which is not averaged. The primary disadvantage of ERPs, as compared to EEG, is that the protocols for their elicitation are more complex. EEG can be acquired with no special stimulation of the user, whereas ERPs can only be acquired when the user is stimulated in a specific and well-controlled manner.

\subsection{Publicly Available Databases}

Instead of collecting live EEG data from human subjects, many prior brain biometric studies in the literature have used and relied on publicly available EEG databases. Development and testing of a robust brain biometric method require databases of sufficient sample size and carefully controlled protocols. In this section, we will review several representative EEG databases and describe the characteristics and contents of each (Table 4).

4.2.1 Alcoholism Database. The Alcoholism Database ${ }^{5}$ [187] studies the correlates of genetic predisposition to alcoholism in terms of EEG activity. The data were measured using a cap containing 64 electrodes, with a sampling rate of $256 \mathrm{~Hz}$. One hundred twenty-two human subjects, including both alcoholics and controls, were presented with the Snodgrass and Vanderwart picture

\footnotetext{
${ }^{5}$ Available online at https://archive.ics.uci.edu/ml/datasets/EEG+Database.
} 
set [154], one or two pictures at a time. When two pictures were shown, they could be the same or different ones. Each subject completed 120 trials where different stimuli were shown. There are three versions of the dataset available: (i) the Small Data Set, containing data for 2 subjects, one alcoholic and the other control; (ii) the Large Data Set, containing data for 10 alcoholic subjects and 10 control subjects; and (iii) the Full Data Set, containing data for all 122 subjects.

4.2.2 ATR Dataset. The ATR dataset ${ }^{6}$ [104] recorded EEG signals from 51 participants using 64 channels at a sampling rate of $256 \mathrm{~Hz}$. Each subject was guided to perform one 5-minute restingstate session first and followed by another session of 8 tasks. Each task session had 24 blocks, where each block included three phases: a 6- to 10-second rest phase, 4-second control phase, and 8 -second attention phase. The subjects were asked to covertly attend to left or right without eye movement according to the visual stimulus cues.

4.2.3 DEAP. DEAP ${ }^{7}[72]$ is a multimodal database for human emotion analysis. The database is composed of recordings from 32 participants and includes 32-channel EEG, Galvanic Skin Response (GSR), respiration amplitude, skin temperature, electrocardiogram (ECG), Blood Volume Pressure (BVP) by plethysmograph, electromyogram (EMG) of the zygomaticus and trapezius muscles, electrooculogram (EOG), and 22 participants' face videos. During the experiments, each participant performed 40 trials by being presented with 40 1-minute excerpts of music videos selected through a web-based subjective assessment experiment. Each trial consisted of four steps: a 2-second screen displaying the current trial number for progress indication, a 5-second baseline recording, a 1-minute display of the music video, and, finally, the participant's self-assessment for arousal, valence, liking, dominance, and familiarity.

4.2.4 EEG Motor Movement/Imagery Database (EEGMMIDB). The EEG Motor Movement/ Imagery Database ${ }^{8}$ comprises EEG recordings obtained from 109 volunteers on 64 channels using the BCI2000 instrumentation system [150]. It is available in PhysioNet [41]. All subjects participated in 14 experimental runs, including 6 types of tasks: 2 resting-state tasks, 2 motor movement tasks, and 2 imagery tasks. At the very beginning, two 1-minute resting-state tasks, one with eyes open and the other with eyes closed, were recorded. Then, four types of motor movement and imagery tasks were performed in 2-minute runs in the order of opening and closing left or right fist, imagining opening and closing left or right fist, opening and closing both fists or both feet, and imagining opening and closing both fists or both feet according to a target's position on the screen (left, right, top, or bottom). These four runs were repeated three times. All the experimental runs formed the dataset of more than 1,500 1- and 2-minute EEG segments.

4.2.5 BCl Competition Datasets. The BCI competitions, with the goal of testing and evaluating brain signal processing and classification methods for Brain-Computer Interfaces (BCIs), have been held four times (2001 [148], 2003 [13], 2005 [14], 2008 [163]). The first competition website is unaccessible; the other three are available, and the last two have been used in EEG biometric researche.

BCI Competition 2005, dataset IIIa ${ }^{9}[151,171]$, contains EEG data from 3 subjects and 60 channels at a sampling rate of $250 \mathrm{~Hz}$ with a bandpass filter in the range of 1 to $50 \mathrm{~Hz}$ and notch filter on. Each subject performed 60 trials on the tasks of motor imagery of left hand, right hand, foot, and tongue according to the cue of arrow directions displayed on the screen.

\footnotetext{
${ }^{6}$ Available online at http://biomark00.atr.jp/modules/xoonips/listitem.php?indexid=181.

${ }^{7}$ Available online at http://www.eecs.qmul.ac.uk/mmv/datasets/deap/index.html.

${ }^{8}$ Available online at http://www.physionet.org/pn4/eegmmidb/.

${ }^{9}$ Available online at http://www.bbci.de/competition/iii/.
} 
BCI Competition 2008, Graz dataset $\mathrm{A}^{10}$ [17], recorded EEGs from 9 subjects with a sampling frequency of $250 \mathrm{~Hz}$. Besides the $22 \mathrm{EEG}$ channels, 3 EOG channels were also recorded for the purpose of artifact removal sampled at the same frequency with a bandpass filter set between $0.5 \mathrm{~Hz}$ and $100 \mathrm{~Hz}$ and a notch filter of $50 \mathrm{~Hz}$. The eye movement artifacts were particularly assessed at the beginning of each session, where data from 2 minutes of eyes open, 1 minute of eyes closed, and 1 minute of eye movements were recorded. Each subject was instructed to complete two sessions of recordings on different days. Each session had six runs, and each run included four cue-based motor imagery tasks (12 trials for each task): movement imagination of the left hand, right hand, both feet, and tongue.

BCI Competition 2008, Graz dataset $\mathrm{B}^{11}$ [85], consists of EEG recordings from 9 subjects. It used the same sampling rate, filters, and EOG recording strategy as the Graz dataset A. The differences are the channels for recording, imagery tasks, and session numbers. Three channels, $\mathrm{C} 3, \mathrm{Cz}$, and C4, were adopted. All subjects completed 5 sessions in two separate days. In each session, there were six runs of 10 trials of two types of motor imagery tasks (i.e., left hand and right hand movements).

4.2.6 Keirn \& Aunon's Database. The Keirn \& Aunon's Database ${ }^{12}$ [68] was motivated to find brainwave changes in morphology and spectral density across different hemispheres and electrodes. Electrodes were placed on six different locations of scalp (i.e., O1, O2, P3, P4, C3, and C4) with an additional electrode for EOG recording. Five subjects were instructed to perform 5 mental tasks without overt movements in two sessions on separate weeks. The 5 distinct mental tasks were (i) resting with both eyes opened and closed for baseline measurements; (ii) solving nontrivial multiplication problems for which immediate answers were not apparent silently and without movement; (iii) mentally visualizing the rotations about one axis of the complex 3-D block figure learned 30 seconds earlier; (iv) mentally and silently composing a letter to a friend or relative; and (v) silently imagining writing numbers on an imagined blackboard sequentially after the previous one was erased. Since each task had both open- and closed-eyes conditions, 10 different experimental conditions were performed. Each tasks lasted for 10 seconds. The EEG data were sampled at $250 \mathrm{~Hz}$ with a hardware bandpass filter of between 0.1 and $100 \mathrm{~Hz}$.

4.2.7 BNCI Horizon 2020 Project Database. The BNCI Horizon 2020 Project Database ${ }^{13}$ included several datasets, and the Two-class Motor Imagery (Two-class MI) dataset [156] was adopted in the literature for biometric purpose. It recorded 14 subjects using 7 electrodes $(\mathrm{C} 3, \mathrm{Cz}$, and $\mathrm{C} 4$ at the central region and 4 additional electrodes around the center) at a sampling rate of $512 \mathrm{~Hz}$. Participants were instructed to perform 5-second kinesthetic motor imagery of the right hand and of the feet. There was only one session for data recording, with 8 runs and 20 trials for each run.

\section{PREPROCESSING AND POSTPROCESSING}

The raw EEG is typically contaminated with electrical artifacts. The most common of these are $50 / 60 \mathrm{~Hz}$ noises from nearby electronics and muscular artifacts from the movements of the face and eyes. It is typical to preprocess the EEG to reduce or remove these artifacts in order to improve signal quality. Artifacts can be either rejected outright based on some criterion or corrected. We next discuss common methods of artifact rejection and correction.

\footnotetext{
${ }^{10}$ Available online at http://www.bbci.de/competition/iv/.

${ }^{11}$ Available online at http://www.bbci.de/competition/iv/.

${ }^{12}$ Available online at http://www.cs.colostate.edu/eeg/main/data/1989_Keirn_and_Aunon.

${ }^{13}$ Available online at http://bnci-horizon-2020.eu/database/data-sets.
} 
Table 5. Description of EEG Subbands

\begin{tabular}{|c|c|c|}
\hline Name & Frequency $(\mathrm{Hz})$ & Description \\
\hline Delta & $0.5-4$ & $\begin{array}{l}\text { predominant oscillatory activity during deep or slow wave sleep } \\
\text { related to attention to internal processing }\end{array}$ \\
\hline Theta & $4-8$ & related to memory demands \\
\hline Alpha & $8-14$ & $\begin{array}{l}\text { most dominant rhythm which emerges in normal subjects, most } \\
\text { pronounced in the parieto-occipital region }\end{array}$ \\
\hline Beta & $14-30$ & consciously perceived stimuli of alertness and focused attention \\
\hline Gamma & $>30$ & $\begin{array}{l}\text { transient functional integration of neural activity across brain } \\
\text { areas }\end{array}$ \\
\hline
\end{tabular}

\subsection{Artifact Rejection}

The simplest criterion-based method of artifact rejection is to discard data that exceed some voltage thresholds. Eye movements and blink contamination are pervasive problems in ERP research [63]. Muscular artifacts are typically orders of magnitude larger than cortical activity (e.g., a blink artifact may be on the order of $200 \mathrm{uV}$ while an ERP effect may be on the order of $4 \mathrm{uV}$ ), so thresholds of around $100 \mathrm{uV}$ are often used [16, 109, 116, 120,121,136]. The threshold was also considered to be sensitive to electrode location and sensor type. In Chen et al. [24], the threshold was set to $75 \mathrm{uV}$ for the wet electrodes placed in the frontal region and $150 \mathrm{uV}$ for all the dry electrodes. Threshold rejection procedures are relatively crude, however, and more sophisticated rejection procedures, such as those based on a peak-to-peak window or on the slope across time points, are preferred [90].

\subsection{Artifact Correction: Filters}

Powerline noise is caused by the AC power supply. Thus, a notch filter which is a band-stop filter with a narrow stopband at $50 \mathrm{~Hz}$ or $60 \mathrm{~Hz}$ depending on the $\mathrm{AC}$ frequency, was adopted to remove powerline noise [1, 36, 52, 105, 105, 138, 147].

Moreover, useful brain activities are primarily in the lower frequency ranges (see Table 5). As summarized in Campisi and La Rocca[19], the delta wave is a predominant oscillatory activity in the frequency range of $0.5-4 \mathrm{~Hz}$ during deep or slow wave sleep and is related to attention to internal processing; the theta wave is in the range of $4-8 \mathrm{~Hz}$ and more related to memory access; the alpha wave in the frequency band of $8-14 \mathrm{~Hz}$ is more likely to be generated in the parietooccipital region during relaxation with eyes closed; the beta band in $14-30 \mathrm{~Hz}$ is related to consciously perceived stimuli; and the gamma band is in the frequency range over $30 \mathrm{~Hz}$ and is related to the transient functional integration of neural activity across brain areas. Different brain tasks can be reflected in different brain rhythms. Therefore, removing less related brain activities can improve the signal quality, and various filters can be an effective way to keep the most relevant information. Low-pass filters of $40 \mathrm{~Hz}$ [24, 177, 178], $41 \mathrm{~Hz}$ [95, 96], and $50 \mathrm{~Hz}$ [81], or bandpass filters of $0.01-50 \mathrm{~Hz}$ [27], $0.01-100 \mathrm{~Hz}$ [31], $0.1-42 \mathrm{~Hz}$ [172], $0.1-55 \mathrm{~Hz}$ [98], $0.1-100 \mathrm{~Hz}$ [36], 0.5-8 Hz [33], $0.5-30 \mathrm{~Hz}[78,79], 0.5-40 \mathrm{~Hz}[114,130], 0.5-45 \mathrm{~Hz}$ [168], $0.5-50 \mathrm{~Hz}$ [9], $0.5-70 \mathrm{~Hz}$ [138, 147], $1-$ $30 \mathrm{~Hz}$ [133], 1-50 Hz [143], 2-47 Hz [74], 2-50 Hz [65, 83], 2-55 Hz [64], 4-52 Hz [4], 5-100 Hz [50, 51], $8-30 \mathrm{~Hz}[52,54,92,162], 14-50 \mathrm{~Hz}$ [53], 20-50 Hz [120], and so on, were implemented to keep the data within a specific frequency range. The Butterworth filter, which has the maximally flat magnitude in the passband, is also popular for EEG preprocessing. The 10th-order Butterworth filter in the $3 \mathrm{~dB}$ passband of $30-50 \mathrm{~Hz}[116,121,136,188]$ was a popular setting, while the 5th-order of $6-35 \mathrm{~Hz}$ Butterworth filter [76], the 3rd-order of $1-50 \mathrm{~Hz}$ [124], and the 4th-order of $0.5-30 \mathrm{~Hz}$ 
[106] were also adopted. Elliptic filters have equalized ripple (equiripple) behavior in both the passband and the stopband. Abo-Zahhad et al. [2] utilized the 3rd-order elliptic bandpass filter with a pass-band ripple of $0.1 \mathrm{~dB}$ and stop-band attenuation of $50 \mathrm{~dB}$. Ashby et al. [7] used a 2nd-order $2 \mathrm{~Hz}$ cutoff elliptic high-pass filter. And Palaniappan [117] adopted an elliptic Finite Impulse Response (FIR) filter of $0.5 \mathrm{~Hz}$ highpass, $30 \mathrm{~dB}$ minimum attenuation in the stop-band, and $0.5 \mathrm{~dB}$ ripple in the pass-band. The surface Laplacian filter, which filters out spatially broad features, was adopted by Marcel and Millán [100].

\subsection{Artifact Correction: Independent Component Analysis}

Independent Component Analysis (ICA) is a Blind Source Separation (BSS) method used to find individual source signals from mixed multidimensional data. Given the mixed signal matrix $X$, with each row representing channels and each column representing the time points, ICA finds the component matrix $W$ to unmix the original data $X$ and get the source matrix $U$ by solving the problem of $U=W X$. It has been demonstrated that ICA can decompose multichannel EEG signals into different components, including EOG, ECG, EMG, different frequency bands, and ERP [115]. Thus, ICA has been widely adopted in the preprocessing stage for artifacts removal $[8,35,50,51$, $89,174]$.

\subsection{Artifact Correction: Principal Component Analysis}

Principal Component Analysis (PCA) is a statistical technique that can convert a set of correlated observations into linearly uncorrelated orthogonal components. Since the first few principal components represent most information of the original data, PCA is implemented to remove noise and increase the SNR by ignoring the less important components and to reconstruct the input signal using the first few components $[121,136]$.

\subsection{Postprocessing}

The temporal nature of the ERP signals means that the epochs from the same person appear consecutively. Currently used machine learning algorithms (e.g., NN, SVM, etc.) are all static classifiers, which means that they are memoryless classifiers and do not consider timing. However, classifiers that take time into account, like Recurrent Neural Network (RNN), Time Delay Neural Network (TDNN), or reservoir computing, have complex training procedures and thus can not always converge properly. To resolve this issue, a postprocessing strategy was proposed [37] which remembered past output labels and used these labels and current output to correct those elements thought to be unreasonable outputs. Results showed that postprocessing can improve accuracy.

\section{FEATURE EXTRACTION}

Feature extraction represents one of the most important stages in the processing and analysis of EEG signals because how well the extracted features represent the EEG signals influences the performance of the recognition system. The adopted features can be classified into groups of domains (i.e., time domain, frequency domain, and time-frequency domain) or channels (i.e., single-channel and two-channel). Here, the widely used features in time domain, frequency domain, and timefrequency domain are described.

\subsection{Time Domain}

The Autoregressive (AR) model is a widely used time-domain feature in EEG biometrics. The AR model represents a type of random process by specifying that the output variable depends linearly on its own previous values and on a stochastic term (an imperfectly predictable term). Denoting that $x(n)$ is the current values of one channel, $a_{i}$ is the AR coefficients at delay $i, e(n)$ is the error 
at time $n$, and $p$ is the order, the AR model is shown in Equation (1):

$$
x(n)=-\sum_{i=1}^{p} a_{i} x(n-i)+e(n) .
$$

Compared to the Yule-Walker method, which applies a pth order AR model to the windowed input signal by minimizing the forward prediction least square error and solving the autoregressive parameters directly, Burg's method was selected much more frequently for its lower computational complexity [122]. It estimates the parameters using the Levinson-Durbin algorithm based on the last autoregressive-parameter estimated from each model order $p$ by minimizing the forward and backward prediction error. In order to determine the optimal order of the AR model, there are generally three methods: minimizing the error of the predictor equation through experimental results with different orders, minimizing the Akaike Information Criterion (AIC), and based on the eigenvalues of the matrix $\tilde{R}$ in the Yule-Walker equations [5, 54, 69, 118].

Although AR is a popular method to extract EEG feature, it can only fit into one time-series data stream. However, sometimes the prediction not only depends on the historical trend of the same time series, but also the previous data of other time series. Thus, a multivariate AR (mAR) model [50] was proposed. Defining that $N$ is the total number of channels, $x_{c}(n)$ represents the current values of channel $c$ where $1 \leq c \leq N$, $p$ is the model order, $a_{c i}$ is the mAR coefficient at delay $i$ for channel $c$, and $e_{c}(n)$ is the error at time $n$ of channel $c$, the mAR model is shown in Equation (2). From this equation, the calculation of the present value of one channel involves all the history of all the channels. Thus mAR can find information on interactions among brain regions and enhance performance:

$$
x_{c}(n)=\sum_{i=1}^{p} a_{c 1}(i) x_{1}(n-i)+\cdots+\sum_{i=1}^{p} a_{c N}(i) x_{N}(n-i)+e_{c}(n) .
$$

A bilinear model used by Poulos et al. [132] is another extension of the AR model. The difference is that it has extra bilinear components $\{x(n-i) e(n-j)\}$ with coefficients $b_{i j}$ and order $k$ and $m$ as shown in Equation (3):

$$
x(n)+\sum_{i=1}^{p} a_{i} x(n-i)=\sum_{i=1}^{k} \sum_{j=1}^{m} b_{i j} x(n-i) e(n-j)+e(n) .
$$

\subsection{Frequency Domain}

As described in Section 5.2, EEG signals can be separated into different frequency bands which are related to various brain activities. Converting EEG data into the frequency domain can extract and distinguish the dominant frequency components.

Power Spectral Density (PSD) describes the signal strength distribution in the frequency domain. Fourier Transform (FT) is an effective way to transform the EEG signal from the time domain into the frequency domain. Based on the PSD obtained through squaring of the absolute value of Fourier-transformed data in each segment, the concavity of spectral distribution [107], variance of spectral power, and the nondominant region of the power spectrum [102] were calculated as EEG features for further recognition purpose. Fractional Fourier Transform (FRFT), seeking onedimensional time-frequency distribution, was used to extract fractional spectral coefficient values for each segment of normalized EEG data [139].

In addition to the FT method, Welch's method is another popular algorithm to estimate the PSD. Welch's method first splits the input signals into overlapping segments and then, based on the segments, the periodogram is calculated by the squared magnitude of the Discrete Fourier 
Transform (DFT) results. The individual periodograms are averaged to reduce the variance of the individual power measurements. This method has been adopted in several studies [112, 127, 128].

Spectral Power (SP) and energy are another two frequency domain features based on the PSD. They are calculated using Parseval's Theorem. Other features based on PSD (e.g., maximum power and frequency in alpha band) were also adopted. Spectral coherence connectivity, which is estimated by coherence, was also used [81] to quantify the level of synchrony between two stationary signals at a specific frequency. Interhemisphere Power Difference (IHPD) and Interhemispheric Channel Linear Complexity (IHLC) values, which are the entropy of the eigenvalues of the covariance matrix of the IHPD and measure the degree of spatial synchronization of data, were also features on the basis of the PSD [7].

\subsection{Time-frequency Domain}

Wavelet Packet Decomposition (WPD) is a wavelet transform algorithm providing both time and frequency information on the signals. In WPD, the signal passes through more filters to build the complete wavelet packet tree, with both the detail and approximation coefficients being decomposed, and thus it is more robust than Discrete Wavelet Transform (DWT) where only the previous approximation coefficients are used to pass through quadrature mirror filters. Cai et al. [18] decomposed the EEG signals using 6-level Daubechies 4 (DB4) WPD and extracted the mean energy and variance of each subband as features. Kumari and Vaish [76] also used 5-level DB4 WPD to extract the subband features of mean, standard deviation, and energy. Gui et al. [43] used 4-level WPD to separate the EEG signals into 5 subbands, including delta, theta, alpha, beta, and gamma, and then used the mean, standard deviation, and entropy as the features. Morlet wavelets [80, 114, 174] were used to transform the EEG signal into the time-frequency domain because this wavelet has a symmetrical and smooth Gaussian shape in both the time and frequency domains. Bump modeling was adopted to reduce the parameter size of a time-frequency map.

\subsection{Others}

In addition to the frequently used features just discussed, other features have also been studied. The EEG signal is approximately stationary in a short period of time between $5 \mathrm{~ms}$ and $100 \mathrm{~ms}$. But over a long time period, the signal is nonstationary. The signals change to reflect different brain activities. Based on this "quasi-stationarity," which is also observed in speech signals, features used in speech signals, like Mel-Frequency Cepstral Coefficients (MFCC), energy, pitch, zero crossing measure, probability of voicing, jitter, and shimmer, were extracted for EEG-based biometric classification [113]. Ma et al. [91] extracted spectrum topographies so that the features were image-based and the biometric recognition problem was converted to image classification problem. Other features were also explored in various studies, like skewness and kurtosis [147], two-channel features of Mutual Information (MI) [138, 147] and Coherence (COH) [87, 138, 147], and so on. More features are summarized in tables in Section 8.

\section{CHANNEL/FEATURE SELECTION AND DIMENSIONALITY REDUCTION}

Although many studies on EEG biometrics directly feed the extracted features to the classifiers for decision-making, channel selection, feature selection, and feature dimensionality reduction are also important for improving the performance and computational efficiency of biometric recognition systems. Data captured by a large number of channels or too many extracted features-the extracted feature dimension can easily reach over thousands-can contain a high degree of irrelevant and redundant information which may greatly degrade the performance of learning algorithms [183, 184]. Furthermore, this could result in a significant challenge in computational complexity, especially for real-time recognition. Nowadays, EEG sensing is more likely to use wearable 
devices for raw data acquisition and wireless data transmission, even for web-based applications [30]. These could lead to another limitation in terms of data transmission rate. Thus, selecting most relevant data by using more effective channels, more representative features, or containing most of the feature information but compressing it to a lower dimension are possible solutions. It is worth noting that some papers use the term "feature selection" even though the actual selection operation is to use features to choose those channels with best performance. Hence, here we categorize these as "channel selection." Only those scenarios involving the addition or removal of features without channel selection are considered "feature selection."

\subsection{Channel Selection}

Placing EEG electrodes onto the most effective brain region according to the testing protocols or on selected places from different regions is an efficient way to use fewer channels. For example, the occipital lobe is the visual processing center of the human brain; thus EEG signals collected from this region may give better results than others when doing VEP biometric recognition tasks.

But it can happen that the selected channels could be unstable, with bad signal quality or even no signal due to noise, movements, disconnections, and many other reasons. In those cases, more channels can provide more reliable and robust data collection. Altahat et al. [4] selected channels according to their stability values, defined as the difference between the "within person distance" (intra-subject distance, distance distribution of the feature vectors for the same person, same channel and same mental task) and the "between person distance" (inter-subject distance, distance distribution of the feature vectors for the same channel and same mental task, but different persons). Using a sequential forward selection algorithm, the process starts with the channel having the highest stability value to get authentication accuracy, then sequentially includes the next highest channel. If the newly added channel does not improve performance by a set threshold, it is removed since the channel feature is redundant. This procedure ends when all channels have been tested. Then the final retained channel set is considered the optimal selection. The result suggested that 8 channels is sufficient to reach the competitive HTER instead of using all 64 channels. Li et al. [86] also used the intra-subject and inter-subject strategy based on Euclidean distance to select 8 channels from 32 EEG channels and 8 physiological channels and reached an identification accuracy of $94 \%$. Another statistical channel selection method, particularly used in authentication scenarios with self-face and non-self-face visual stimuli, was the pointwise biserial correlation coefficient. With the selected 5 or 6 channels, authentication accuracy was up to $92.4 \%[177,178,182]$.

SVM Recursive Feature Elimination (SVM-RFE) is another technique for feature selection. It finds the feature ranking list through iterations based on the following steps. First, the weight vector of dimension length of surviving features is computed from the SVM training model on the surviving features. Then the ranking criteria is computed. The feature with smallest ranking criterion is found, and the feature rank list is updated. The feature with smallest ranking criterion is eliminated, and the new surviving feature subset is formed. Moreover, SVM-RFE can be generalized to remove more than one feature per step to increase its speed [47]. Liu et al. [89] and Bai et al. [8] used SVM-RFE to select the most effective channels. Results showed that the selected 32 channels obtained a higher identification accuracy of $97.25 \%$ than the $96.25 \%$ reached by using all 64 channels.

The Genetic Algorithm (GA) is an adaptive, probabilistic search method for global optimization originating in biological natural selection. It consists of three basic operations: selection, crossover. and mutation. During the "selection" stage, the channel features with the highest accuracy were selected to breed a new generation. "Crossover" represented the analogous process to mating in a biological system and formed new offspring by varying the programming of a chromosome or 
chromosomes from one generation to the next. Part of the new individuals were created through the "mutation" process, analogous to biological mutation, which randomly changed the allele values in the chromosome and helped maintain genetic diversity from one generation of a population to the next. When a new generation of individuals was produced through these three operations, the process is repeated until a termination condition is fulfilled, such as when the iteration process reaches a pre-set generation or when the fitness metric no longer rises. Bai et al. [8] applied this algorithm to optimize channels, and the classification accuracy was improved from $96.25 \%$ to $97 \%$ by reducing the number of channels from 64 to 37.

The Fisher Discriminant Ratio (FDR) was adopted to evaluate the discrimination level of features and to select the most discriminatory features [8]. Based on such evaluations, it was shown that there was no optimization on the channel number, which was still all 64 channels, and therefore the accuracy of $96.25 \%$ was not changed.

The Davies-Bouldin Index (DBI) evaluates feature importance by the number of clusters in the data and was adopted in Palaniappan and Mandic [120] to select the more representative features to optimize recognition accuracy since, for each channel, only the gamma band energy was extracted and each channel had one feature value. Therefore, sorting the DBI of the features from the 61 channels indicated the importance of the channel. Using this method, the optimal number of channels was 35, with a GAR of $98.56 \%$ compared to using all 61 channels with about $99 \%$ accuracy.

The Flower pollination algorithm [180] is a global optimization method motivated by the pollination process of flowers based on four rules: (i) biotic cross-pollination is the global pollination, and Lévy flights constrain pollen-carrying movements; (ii) abiotic pollination and self-pollination are the local pollination; (iii) pollinators contribute to flower constancy; and (iv) interaction of local pollination and global pollination is controlled by a probability between 0 and 1 and slightly biased to local pollination. The binary flower pollination algorithm was adopted in Rodrigues et al. [140], where the search space is binary instead of continuous, and it selected less than half of 64 channels but maintained a recognition accuracy of up to $87 \%$.

A rough set is a method to learn data dependence and evaluate the quality of a feature subset. Rough set neighbor and rough set entropy were adopted in Moreno-Revelo et al. [103] and selected 12 more relevant channels out of 32 EEG channels and 8 peripheral channels for person identification. The best accuracy could reach $100 \%$.

\subsection{Feature Selection}

More often, the number of electrode channels used is small, and it is thus less persuasive to select one or a few channels. The final result will largely rely on the features extracted from the raw signals. It is thus imperative to select the most representative features.

Linear Discriminative Analysis (LDA) is a method based on intra-class and inter-class means to find those directions having the maximum linear separability of classes. The highest recognition accuracy was achieved when using LDA on the optimized Eigenbrains (EBs) and Eigentensorbrains (ETBs) after discriminative analysis [96]. It was also mentioned by Cai et al. [18] that using LDA can reduce feature dimensionality. However, no further details, like performance, were presented. Considering one feature of one channel as a feature attribute, Lee et al. [83] used intra- and intersubject mutual information to select features. Out of 112 features (7 features $\times 16$ channels), 9 features were selected to achieve an identification accuracy of $95 \%$.

One-Way Analysis of Variance (ANOVA) is a statistic model to analyze the differences of means and variance among classes. Thus it can be applied to explore the intra-class and inter-class variance of features. La Rocca et al. [80] used this method to select more discriminant parameters and shrank feature size from 39 to 13 . 


\subsection{Dimensionality Reduction}

PCA linearly transforms a set of variables into a new set of orthogonal variables called principal components in which most data information is retained in the first few principle components. Wang and Najafizadeh [174] used PCA to identify the most relevant 48 channels from 128 channels. The identification accuracy was $53.5 \%-99.73 \%$, compared to $51.09 \%-99.14 \%$ when using all 128 channels. Palaniappan [117] used PCA to reduce the feature dimensions from 126 to 11 while retaining $99.99 \%$ of the variance. Koike-Akino et al. [73] selected 100 PCA components out of 1,442 feature dimensions to maintain $90 \%$ of the data variance, as well as using the partial least-squares method to explain $65 \%$ of the variance using 100 components. Falzon et al. [36] used 15 principle components to retain $90 \%$ of the total variance. PCA and Multilinear PCA (MPCA) were used in brain biometric recognition [95, 96], as well as for PSD feature projection (EB and ETB) [96].

The Johnson-Lindenstrauss Transform used in $\mathrm{He}$ et al. [50] is also a robust and time-efficient dimensionality reduction method. It is based on the lemma that $n$ points in the Euclidean space can be projected down to the dimension of $O\left(\epsilon^{-2} \log (n)\right)$ with a distortion within the range of $1 \pm \epsilon(0<\epsilon<1)$, in the pairwise distance. Using this transform, the feature size was reduced from $256 \times 4$ to $100 \times 4$.

\section{CLASSIFICATION}

When evaluating the performance of a specific biometric approach, multiple metrics were proposed and adopted [19], including the Correct Recognition Rate (CRR) in the identification case, False Acceptance Rate (FAR), False Rejection Rate (FRR), Genuine Authentication Rate (GAR), Half Total Error Rate $(\mathrm{HTER}=(\mathrm{FAR}+\mathrm{FRR}) / 2)$, and Equal Error Rate (EER), which is the HTER when FAR=FRR in the authentication case. Moreover, the CRR in this survey also referred to the accuracy in authentication scenarios. In some papers, chance accuracy was used, and it was calculated based on predefined confidence probabilities according to similarity ranks, considering that the correct labels were not in the first rank but very close to it [144].

\subsection{Similarity-Based Pattern Recognition}

Similarity-based pattern recognition is a straightforward classification category to authenticate or identify individuals based on the selected similarity evaluation metrics [126]. For the authentication case, during enrollment, the system identifies both intra- and inter-class similarity ranges and then finds a proper threshold based on similarity values to separate the specific person from others. But for the identification case, the classification results are not simply acceptance or rejection by using a threshold. K-Nearest Neighbors $(\mathrm{kNN})$, which make final decisions by majority rule on the closest or most similar points to the inputs, were usually selected for identification. Specifically, prior studies tried different similarity metrics in $\mathrm{kNN}$ for recognition, as summarized in Table 6. If the similarity metric was not explicitly specified in the paper, it was marked as "kNN." Commonly used similarity measurements, like Cross-Correlation (CC) [6, 142-146, 167, 168, 172, 173], Cosine Similarity [28, 32, 62, 95-97, 106], Euclidean Distance [9, 10, 16, 39, 42, 44, 64, 9597, 109, 121, 124, 174, 181], Mahalanobis Distance [81, 95, 96, 166, 167], Manhattan Distance [54, 95-97, 117, 119, 121, 130, 136], and Bhattacharyya Distance [83] have been investigated in the literature.

Moreover, it has been acknowledged that ERP patterns often had some sort of unintended misalignment or distortion. Thus Dynamic Time Warping (DTW) [34, 38, 42] was adopted to mitigate this problem. Because the extracted EEG features may not have the contributions equal to the classification results, and the intra-class features may have large variations due to changes in recording conditions, it is difficult for the regular Euclidean distance-based $\mathrm{kNN}$ to provide 
Table 6. Performance of Similarity-Based Methods

\begin{tabular}{|c|c|c|c|c|c|c|}
\hline Papers & Sbjs & Chs & Protocols & Features & Similarity & Performance \\
\hline$[6]$ & 30 & 1 & ERP & raw ERP & $\mathrm{CC}$ & Chance accuracy: $92 \%$ \\
\hline [9] & 9 & 61 & $\mathrm{RC}$ & peaks & Euclidean & EER: $11.17 \%$ \\
\hline [10] & 52 & 64 & $\mathrm{RC}, \mathrm{RO}$, Mental & PSD & Euclidean & EER: $22.97 \%-29.36 \%$ \\
\hline [16] & $\begin{array}{l}120 \\
6\end{array}$ & $\begin{array}{l}64 \\
106\end{array}$ & $\begin{array}{l}\text { ERP } \\
\text { Mental }\end{array}$ & $\mathrm{AR}$ & Euclidean & $\begin{array}{l}\text { CRR: } 93.24 \%-96.34 \% \\
\text { CRR: } 98.52 \%-99.41 \%\end{array}$ \\
\hline [18] & $40,33,11$ & 1 & $\mathrm{RC}$ & AR, PSD, WPT & $\begin{array}{l}\text { kNN } \\
\text { L-LMNN }\end{array}$ & $\begin{array}{l}\text { CRR: } 50.99 \%-93.94 \% \\
\text { CRR: } 58.47 \%-98.03 \%\end{array}$ \\
\hline [28] & 15 & 1 & RC, Mental & PS & Cosine & $\begin{array}{l}\text { HTER: } 1.1 \%-43.3 \% \\
\text { CRR: } 22 \%\end{array}$ \\
\hline [31] & 20 & 20 & ERP & LDA & $\mathrm{kNN}$ & CRR: $97 \%$ \\
\hline [32] & 50 & 6,17 & ERP & raw ERP & Cosine & EER: up to $\sim 22 \%$ \\
\hline [34] & 50 & 6,17 & Mental & raw EEG & DTW & EER:7\%-25\% \\
\hline [36] & 8 & 32 & ERP & variance & $\mathrm{kNN}$ & $\begin{array}{l}\text { GAR: } 91.7 \% \\
\text { FAR: } 1 \%\end{array}$ \\
\hline [38] & 12 & 1 & Mental & $\begin{array}{l}\text { MPA, MSP, MSV, } \\
\text { PF, PFM, SP, } \\
\text { VAZ, ZCR }\end{array}$ & DTW & EER: $14.7 \%-67.6 \%$ \\
\hline [39] & 109 & 64 & $\mathrm{RC}, \mathrm{RO}$ & nodal EC & Euclidean & EER: up to $14.4 \%$ \\
\hline [42] & 30 & 1 & ERP & raw ERP & $\begin{array}{l}\text { Euclidean } \\
\text { DTW }\end{array}$ & $\begin{array}{l}\text { CRR: } 53.33 \%-81.17 \% \\
\text { CRR: } 33.83 \%-67.17 \%\end{array}$ \\
\hline [44] & 37 & 1 & ERP & raw ERP & Euclidean & CRR: $47.3 \%-67.57 \%$ \\
\hline [54] & 50 & 19 & $\mathrm{RC}, \mathrm{RO}$ & $\mathrm{AR}$ & Manhattan & CRR: up to $95 \%$ \\
\hline [64] & 109 & 34 & $\mathrm{RC}, \mathrm{RO}$ & $\begin{array}{l}\text { EC, MaxLyp, MPC, } \\
\text { PE, PLI, PSD, SE }\end{array}$ & Euclidean & $\begin{array}{l}\text { FRR@1\% FAR: } \\
0.92 \%-2.2 \% \\
\text { EER: } 0.63 \%-1.8 \%\end{array}$ \\
\hline [62] & $15+3 I$ & 1 & RC, Mental & PS & Cosine & HTER: $1 \%$ \\
\hline [81] & 108 & 56 & $\mathrm{RC}, \mathrm{RO}$ & $\mathrm{COH}, \mathrm{PSD}$ & Mahalanobis & CRR: $75.86 \%-90.49 \%$ \\
\hline [83] & 7 & 6 & $\mathrm{RC}$ & PSD & Bhattacharyya & CRR: $95 \%$ \\
\hline [87] & 10 & 10 & ERP & $\mathrm{CC}, \mathrm{COH}, \mathrm{MA}$ & FRNN & CRR: $90.17 \%$ \\
\hline [95] & 60 & 19 & $\mathrm{RC}$ & EB, LDA, PSD & $\begin{array}{l}\text { Cosine, } \\
\text { Euclidean, } \\
\text { Manhattan, } \\
\text { Mahalanobis }\end{array}$ & CRR: up to $97.68 \%$ \\
\hline [96] & 30 & 19 & $\mathrm{RC}, \mathrm{RO}$ & EB, ETB, LDA, PSD & $\begin{array}{l}\text { Cosine, } \\
\text { Euclidean, } \\
\text { Manhattan, } \\
\text { Mahalanobis }\end{array}$ & CRR: up to $90.49 \%$ \\
\hline [97] & 50 & 19 & $\mathrm{RC}, \mathrm{RO}$ & $\mathrm{AR}, \mathrm{COH}, \mathrm{PSD}$ & $\begin{array}{l}\text { Manhattan } \\
\text { Euclidean, } \\
\text { Cosine }\end{array}$ & CRR: up to $\sim 86 \%$ \\
\hline [102] & 23 & 1 & $\mathrm{RC}$ & FFT & User define & GAR: $70 \%-79 \%$ \\
\hline [106] & $15+5 I$ & 2 & $\mathrm{RC}$ & AR, PSD & Cosine & $\begin{array}{l}\text { FAR: } 1 \%-2.3 \% \\
\text { FRR: } 13.7 \%-32.2 \% \\
\text { HTER: } 7.3 \%-17.2 \%\end{array}$ \\
\hline [107] & 23 & 1 & $\mathrm{RC}$ & FFT & User define & EER:11\% \\
\hline [108] & 30 & 1 & $\begin{array}{l}\text { driving scenes, } \\
\text { route tracing }\end{array}$ & PS & User define & EER: $22 \%-52 \%$ \\
\hline [109] & 20 & 14 & ERP & spectral differences & Euclidean & EER: $23 \%-56 \%$ \\
\hline
\end{tabular}

(Continued) 
Table 6. Continued

\begin{tabular}{|c|c|c|c|c|c|c|}
\hline Papers & Sbjs & Chs & Protocols & Features & Similarity & Performance \\
\hline [110] & 23 & 1 & driving scenes & PS & User define & EER: $27 \%-37 \%$ \\
\hline$[117]$ & 5 & 6 & Mental & $\begin{array}{l}\text { AR, IHLC, IHPD, } \\
\text { NLC }\end{array}$ & Manhattan & $\begin{array}{l}\text { FRR: } 0-1.5 \% \\
\text { FAR: } 0\end{array}$ \\
\hline [119] & 102 & 61 & ERP & MUSIC & Manhattan & CRR: $95.00 \%-96.13 \%$ \\
\hline [121] & 20 & 61 & ERP & GBSP & $\begin{array}{l}\text { Euclidean } \\
\text { Manhattan }\end{array}$ & $\begin{array}{l}\text { CRR: } 66.12 \%-91.54 \% \\
\text { CRR: } 65.64 \%-94.18 \%\end{array}$ \\
\hline [124] & 10 & 18 & Motor & $\mathrm{COH}$ & Euclidean & EER: $7.5 \%$ \\
\hline [130] & 25 & 19 & ERP & $\mathrm{AR}, \mathrm{MFCC}$ & Manhattan & CRR: up to $100 \%$ \\
\hline [136] & 20 & 61 & ERP & GBE & Manhattan & CRR: $70.88 \%-92.04 \%$ \\
\hline [142] & 50 & 1 & ERP & raw ERP & $\mathrm{CC}$ & CRR: $60 \%-86 \%$ \\
\hline [143] & 20 & 26 & ERP & raw ERP & $\mathrm{CC}$ & CRR: $100 \%$ \\
\hline [144] & 32 & 1 & ERP & raw ERP & $\mathrm{CC}$ & CRR: $56 \%$ \\
\hline [145] & 32 & 1 & ERP & raw ERP & $\mathrm{CC}$ & Chance accuracy: $90 \%$ \\
\hline [146] & 9 & 1 & ERP & raw ERP & $\mathrm{CC}$ & CRR: up to $100 \%$ \\
\hline$[158-160]$ & 40 & 1 & $\begin{array}{l}\text { RC } \\
\text { (water/coffee) }\end{array}$ & PSD & $\mathrm{kNN}$ & CRR: $93.75 \%-97.5 \%$ \\
\hline [166] & 109 & 64 & $\mathrm{RC}, \mathrm{RO}$ & $\mathrm{AE}, \mathrm{SE}$ & Mahalanobis & CRR: up to $99.7 \%$ \\
\hline$[167]$ & 109 & 19 & $\mathrm{RC}, \mathrm{RO}$ & DBP, IAF, IAFP & $\begin{array}{l}\text { CC, } \\
\text { Mahalobonis }\end{array}$ & CRR: $90.21 \%$ \\
\hline$[168]$ & 4 & 14 & ERP & PSD & $\mathrm{CC}$ & $\begin{array}{l}\text { FAR: } 12.5 \% \\
\text { FRR: } 12.5 \%\end{array}$ \\
\hline [172] & 6 & 19 & ERP & raw ERP & $\mathrm{CC}$ & GAR: $73.33 \%-96.67 \%$ \\
\hline [173] & $4+21$ & 8 & Mental & topological plot & 2-D CC & FAR: $1.01 \%-50.48 \%$ \\
\hline [174] & 4 & 128 & $\mathrm{RC}$ & CWT & Euclidean & CRR: $51.09 \%-99.73 \%$ \\
\hline [181] & 20 & 61 & ERP & AR, PSD & Euclidean & CRR: up to $100 \%$ \\
\hline [188] & 70 & 8 & ERP & Energy & $\mathrm{kNN}$ & $\begin{array}{l}\text { GAR: } 31.3 \%-95.1 \% \\
\text { FAR: } 0.9 \%-6.4 \%\end{array}$ \\
\hline
\end{tabular}

good performance. Thus the Local Large Margin Nearest Neighbor (L-LMNN) [18] was proposed to find the relative importance and correlations of each feature dimension, minimizing the intraclass distance while maximizing inter-class distance. The Fuzzy-Rough Nearest Neighbors (FRNN) [87] algorithm combines fuzzy sets, rough sets, and the nearest neighbor classification. It determines an input test feature class according to its membership value calculated from the fuzzy upper and lower approximations. In addition to these commonly used similarity evaluation metrics, user-defined approaches, in which the similarity was evaluated according to some custom-defined metrics by combining extracted features, were also adopted [102, 107, 108, 110].

\subsection{Support Vector Machine}

An SVM uses a hyperplane to separate two classes of data by maximizing the margin, which is the distance between the nearest training points from different classes, and has a good generalization capabilities. Because the ordinary SVM classifier can only separate two classes, in multiclass problem, there are two ways to implement the multiclass SVM: One-Against-One (OAO) and OneAgainst-All (OAA). OAA separates the data into two groups: a random class as one group and all other classes as the other group. Then the question is converted into a two-class problem. Thus the final decision is made according to the highest output. OAO chooses any two classes for classification. After this, by combining the results from each classifier, the class with the most votes 
Table 7. Performance of SVM Methods

\begin{tabular}{|c|c|c|c|c|c|c|}
\hline Papers & Sbjs & Chs & Protocols & Features & Kernels & Performance \\
\hline [6] & 30 & 1 & ERP & raw ERP & SVM & Chance accuracy: $83 \%$ \\
\hline [7] & 5 & 14 & Mental & $\begin{array}{l}\text { AR, IHPD, IHLC, } \\
\text { PSD, SP }\end{array}$ & Linear & $\begin{array}{l}\text { FRR: } 0-11.3 \% \\
\text { FAR: } 0-2 \% \\
\text { CRR: } 97.69 \%\end{array}$ \\
\hline [8] & 20 & 64 & ERP & AR, FPS, PLV, TPS & SVM & CRR: $88.63 \%-97.25 \%$ \\
\hline [11] & 9 & 5 & $\mathrm{RC}$ & ABS, MSD, WPES, WPS & SVM & GAR: $44.44 \%-94.44 \%$ \\
\hline [16] & $\begin{array}{l}120 \\
6\end{array}$ & $\begin{array}{l}64 \\
106\end{array}$ & $\begin{array}{l}\text { ERP } \\
\text { Mental }\end{array}$ & $\mathrm{AR}$ & Linear & $\begin{array}{l}\text { CRR: } 98.96 \% \\
\text { CRR: } 99.76 \%\end{array}$ \\
\hline [30] & 16 & 1 & $\mathrm{RC}$ & $\begin{array}{l}\text { Energy distribution, } \\
\text { index, PSD }\end{array}$ & $\mathrm{RBF}$ & CRR: $93.73 \%$ \\
\hline [35] & 20 & 128 & video & Cepstral coefficients & Linear & CRR: up to $\sim 99 \%$ \\
\hline [37] & 13 & 20 & ERP & SP & Linear, RBF & Error: $15.67 \%-65.94 \%$ \\
\hline [67] & 60 & 14 & ERP & DB4 wavelets, RMS, std & Linear & CRR: $93.83 \%$ \\
\hline [69] & 104 & 6 & $\mathrm{RC}, \mathrm{RO}$ & $\mathrm{AR}$ & SVM & CRR: $97.43 \%$ \\
\hline [73] & 25 & 14 & ERP & raw ERP & Linear & CRR: 20\%-50\% \\
\hline$[75]$ & $20+30 I$ & 14 & Mental & DFT, mean, std & SVM & $\begin{array}{l}\text { FAR: } 18 \%-59 \% \\
\text { HTER: } 9 \%-29.5 \%\end{array}$ \\
\hline [89] & 20 & 64 & $\mathrm{RC}, \mathrm{ERP}$ & AR, TPS, FPS, PLV & $\mathrm{RBF}$ & CRR: $67.37 \%-96.25 \%$ \\
\hline [106] & $15+5 I$ & 2 & $\mathrm{RC}$ & AR, PSD & $\begin{array}{l}\text { Linear, RBF } \\
\text { Sigmoid } \\
\text { Polynomial }\end{array}$ & $\begin{array}{l}\text { FAR: } 0.3 \%-1.4 \% \\
\text { FRR: } 10.7 \%-50 \% \\
\text { HTER: } 5.5 \%-25.7 \%\end{array}$ \\
\hline$[111]$ & $\begin{array}{l}9 \\
9 \\
40\end{array}$ & $\begin{array}{l}3 \\
3 \\
8\end{array}$ & $\begin{array}{l}\text { Mental } \\
\text { Mental } \\
\text { free }\end{array}$ & AR, PSD & RBF & EER: up to $\sim 37 \%$ \\
\hline [113] & $\begin{array}{l}3,9,9 \\
40 \\
20,122\end{array}$ & $\begin{array}{l}8,8,3 \\
8 \\
8\end{array}$ & $\begin{array}{l}\text { Mental } \\
\text { Free } \\
\text { ERP }\end{array}$ & $\begin{array}{l}\text { Energy, MFCC, Pitch, } \\
\text { probability of voicing, } \\
\text { ZCR, Jitter \& shimmer }\end{array}$ & $\mathrm{RBF}$ & CRR: $46.24 \%-99 \%$ \\
\hline$[127]$ & 9,9 & 3 & Mental & AR, PSD & $\mathrm{RBF}$ & EER: $0-3.3 \%$ \\
\hline [182] & 10 & 18 & ERP & $\begin{array}{l}\text { signal difference, } \\
\text { time derivative }\end{array}$ & $\mathrm{RBF}$ & $\begin{array}{l}\text { GAR: } 86.1 \% \\
\text { FAR: } 13.9 \% \\
\text { FRR: } 13.9 \%\end{array}$ \\
\hline
\end{tabular}

would be the instance classification [37]. Table 7 summarizes brain biometric studies using SVM classifiers. If the kernel was presented as "SVM," then the kernel type was not listed in the paper. Linear kernel is computationally efficient, and it was adopted in several studies $[7,16,35,37,67$, $73,106]$. Sometimes, it is impossible for the linear hyperplane to separate classes. Thus, nonlinear decision boundaries could help in this situation. Nonlinear kernels are introduced to map data to another space to make them more separable while increasing the classifier's complexity. One frequently used kernel is the Radial Basis Function (RBF) [30, 37, 89, 106, 111, 113, 127, 182]. Other kernels, like sigmoid and polynomial, were also tested [106].

\subsection{Neural Network}

The NN, one of the most important and popular machine learning techniques, seeks to find a function that can map inputs to outputs. The classic structure to implement the NN is the multilayer perception NN, which generally has three types of layers: input layer, hidden layer, and output layer. It uses the feedforward and back-propagation algorithm to train the data and calculate the weight matrix. Based on the weight matrix, the result can be predicted. Several papers using NNs to make decisions in brain biometric recognition are listed in Table 8. Many studies used the 
Table 8. Performance of NN Methods

\begin{tabular}{|c|c|c|c|c|c|c|}
\hline Papers & Sbjs & Chs & Protocols & Features & Types & Performance \\
\hline [1] & 10 & $1,2,4$ & $\mathrm{RC}, \mathrm{RO}$ & $\mathrm{AR}$ & DNN & CRR: $70 \%-97 \%$ \\
\hline [27] & 120 & 64 & $\mathrm{RC}, \mathrm{RO}, \mathrm{ERP}$ & raw EEG & $\mathrm{DNN}$ & CRR: $62.2 \%-92.9 \%$ \\
\hline [43] & 32 & 1 & ERP & WPD & $\mathrm{NN}$ & $\begin{array}{l}\text { CRR: up to } 47.5 \% \\
\text { GAR: up to } 94.04 \%\end{array}$ \\
\hline [52] & 15 & 2 & Relax, Mental & PSD & $\mathrm{NN}$ & CRR: $78.6 \%$ \\
\hline [53] & 6 & 2 & Relax, Mental & PSD & NN & CRR: $94.4 \%$ to $97.5 \%$ \\
\hline [55] & $\begin{array}{l}45 \\
122\end{array}$ & 64 & ERP & RMS & $\mathrm{NN}$ & $\begin{array}{l}\text { CRR: } 100 \% \\
\text { CRR: } 95.08 \%\end{array}$ \\
\hline [60] & 20 & 64 & ERP & 1D-LBP, 1D-LGP & $\mathrm{NN}$ & CRR: $62.7 \%, 88.9 \%$ \\
\hline [73] & 25 & 14 & ERP & raw ERP & DNN & CRR: 35\%-65\% \\
\hline [76] & 6 & 14 & Mental & DWT & LVQ & CRR: 90\% \\
\hline [86] & 32 & 32 & ERP & DB4 wavelets & DNN & CRR: $88 \%$ \\
\hline$[105]$ & 10 & 2 & ERP & fuzzy entropy & $\mathrm{NN}$ & $\begin{array}{l}\text { CRR: } 87.3 \% \\
\text { FAR: } 5.5 \% \\
\text { FRR: } 5.6 \%\end{array}$ \\
\hline [116] & 20 & 61 & ERP & GBSP & NN & CRR: $99.06 \%$ \\
\hline [118] & 5 & 19 & ERP & energy & $\mathrm{NN}$ & CRR: $80.6 \%-90 \%$ \\
\hline [119] & 102 & 61 & ERP & MUSIC & ENN & CRR: $96.77 \%-98.12 \%$ \\
\hline [120] & 40 & 61 & ERP & Energy & ENN & CRR: $98.06 \%-98.56 \%$ \\
\hline [121] & 20 & 61 & ERP & GBSP & SFA & CRR: $66.26 \%-92.84 \%$ \\
\hline [131] & $4+75 I$ & 1 & $\mathrm{RC}$ & SP & LVQ & $\begin{array}{l}\text { GAR: } 80 \%-100 \% \\
\text { FAR: } 10 \%-20 \%\end{array}$ \\
\hline [132] & $4+75 I$ & 1 & $\mathrm{RC}$ & AR, bilinear & LVQ & $\begin{array}{l}\text { GAR: } 76 \%-88 \% \\
\text { FAR: } 16 \%-24 \%\end{array}$ \\
\hline [133] & $4+75 I$ & 1 & $\mathrm{RC}$ & AR & LVQ & $\begin{array}{l}\text { GAR: } 72 \%-84 \% \\
\text { FAR: } 18 \%-22 \%\end{array}$ \\
\hline [135] & 14 & $\mathrm{~N} / \mathrm{A}$ & Mental & DB4 wavelets & $\mathrm{NN}$ & CRR: $91.4 \%-93.8 \%$ \\
\hline [136] & 20 & 61 & ERP & Energy & SFA & CRR: $71.14 \%-92.84 \%$ \\
\hline [139] & 7 & 6 & RC, RO, Mental & FRFT & RBNN & GAR: $\sim 80 \%$ \\
\hline [153] & 3 & 4 & $\mathrm{RC}$ & DFT, WPD & $\mathrm{NN}$ & CRR: $87 \%-93 \%$ \\
\hline [162] & 9 & 15 & Mental & Energy & NN & CRR: $92.52 \%, 90.12 \%$ \\
\hline
\end{tabular}

one-hidden layer NN, which was constructed using one input layer, one hidden layer, and one output layer [43, 52, 53, 55, 60, 105, 116, 118, 135, 153, 162]. Besides the conventional one-hiddenlayer NN, the Deep Neural Network (DNN) with two or more hidden layers, was also investigated. Two-hidden-layer NNs with 100 and 1000 nodes on each hidden layer were adopted in Abdullah et al. [1] and Koike-Akino et al. [73] respectively. Four hidden-layer NN was implemented in other work [27, 86], while the output layer in Chu et al. [27] was set to three types: softmax, multiclass SVM, and Random Forest (RF).

In addition to the classic NN, Kohonen's LVQ is another type of NN that has been used during the very early stage of brain biometric studies by Poulos et al. [131-133]. The basic architecture of LVQ has two neuron layers: the competitive layer and the linear layer. It sorts data to the class whose Euclidean distance from the sample vector is smallest, and it has the ability of nonlinear separability. The output function is based on the winner-takes-all principle. Other types of NNs were also adopted, such as the Elman Neural Network (ENN) $[119,120]$ with the addition that the hidden layer outputs are delayed and fed back into the network; the Simplified Fuzzy ARTMAP 
Table 9. Performance of DA Methods

\begin{tabular}{|c|c|c|c|c|c|c|}
\hline Papers & Sbjs & Chs & Protocols & Features & Types & Performance \\
\hline [2] & 31 & 1 & $\begin{array}{l}\text { Eye blink, } \\
\text { RC, ERP }\end{array}$ & AR & LDA & $\begin{array}{l}\text { CRR: up to } 99.68 \% \\
\text { EER: } 0.89 \%-4.4 \%\end{array}$ \\
\hline [24] & 29 & $28 / 16$ & ERP & $\begin{array}{l}\text { point-biserial } \\
\text { correlation coefficients }\end{array}$ & regularized LDA & $\begin{array}{l}\text { GAR: } 100 \% \\
\text { FAR: } 3.33 \times 10^{-3} \%\end{array}$ \\
\hline [61] & 12 & 14 & Mental & CSP & LDA & GAR: up to $96.97 \%$ \\
\hline [73] & 25 & 14 & ERP & raw EPR & LDA, QDA & CRR: up to $96.7 \%$ \\
\hline [74] & $\begin{array}{l}9 \\
14 \\
20 \\
12\end{array}$ & $\begin{array}{l}22 \\
7 \\
16 \\
15\end{array}$ & $\begin{array}{l}\text { Mental } \\
\text { Mental } \\
\text { ERP } \\
\text { Driving }\end{array}$ & $\begin{array}{l}\text { PLV, degree of } \\
\text { brain networks }\end{array}$ & LDA & CRR: $93.7 \%-96.8 \%$ \\
\hline [80] & 36 & 3 & $\mathrm{RC}$ & bump modeling & LDA & CRR: up to $99.69 \%$ \\
\hline [84] & 4 & 1 & $\mathrm{RC}$ & $\alpha$ frequency, MP, SP & LDA & CRR: $86.7 \%-100 \%$ \\
\hline [106] & $15+5 I$ & 2 & $\mathrm{RC}$ & AR, PSD & LDA & $\begin{array}{l}\text { FAR: } 2.3 \%-3.7 \% \\
\text { FRR: } 25.9 \%-44.8 \% \\
\text { HTER: } \\
14.1 \%-24.2 \%\end{array}$ \\
\hline [118] & 5 & 19 & ERP & energy & LDA & CRR: $76.9 \%-93.5 \%$ \\
\hline [121] & 20 & 61 & ERP & GBSP & LDA & CRR: $84 \%-96.5 \%$ \\
\hline [138] & $51+36 \mathrm{I}$ & 2 & $\mathrm{RC}$ & $\mathrm{AR}, \mathrm{CC}, \mathrm{COH}, \mathrm{FT}, \mathrm{MI}$ & $\begin{array}{l}\text { LDA, D-LDA, } \\
\text { QDA, D-QDA }\end{array}$ & EER: $3.4 \%$ \\
\hline [147] & $50+20 \mathrm{I}$ & 2 & $\mathrm{RC}$ & $\begin{array}{l}\mathrm{AR}, \mathrm{CC}, \mathrm{COH}, \mathrm{FT}, \mathrm{MI}, \\
\text { skewness \& kurtosis, } \\
\text { 3rd autocovariance, } \\
\text { ICA, time reversibility, } \\
\text { centroid frequency, } \\
\text { max power frequency }\end{array}$ & $\begin{array}{l}\text { LDA, D-LDA, } \\
\text { QDA, D-QDA }\end{array}$ & $\begin{array}{l}\text { EER: } 2.4 \% \\
\text { CRR: } 93.8 \%\end{array}$ \\
\hline [179] & 108 & 9 & $\begin{array}{l}\mathrm{RC}, \mathrm{RO}, \\
\text { Mental }\end{array}$ & WPD & LDA & $\begin{array}{l}\text { CRR: up to } 99 \% \\
\text { EER: } 4.5 \%\end{array}$ \\
\hline
\end{tabular}

(SFA) $[121,136]$, which consists of a fuzzy ART module for receiving the input features, a category layer with output classes, and an inter=ART module for learning the mapping between input features and corresponding output classes; and the Radial Basis Neural Network (RBNN) [139], which has one hidden radial basis layer and lower training time.

\subsection{Discriminant Analysis}

The aim of Discriminant Analysis (DA), more commonly Linear Discriminant Analysis (LDA), is to use hyperplanes to separate the data of different classes by projecting the data into a lower dimensional space. It maximizes the inter-class distance with the assumption that the data are normally distributed so that the covariance matrices are equal for different classes. Table 9 summarizes studies that adopted DA methods. In addition to LDA [2, 24, 61, 73, 74, 80, 84, 106, 118, 121, 147, 179], other DA variants are available, such as Diagonal Linear Discriminant Analysis (D-LDA), which is similar to LDA but with diagonal covariance matrices; Quadratic Discriminant Analysis (QDA), which fits multivariate normal density with covariance that is not identical for each class; and Diagonal Quadratic Discriminant Analysis (D-QDA), which is similar to QDA but with diagonal covariance matrices $[73,138,147]$.

\subsection{Other Supervised Learning Classification Methods}

In addition to the mentioned frequently adopted classifiers, other supervised learning classification methods for person recognition have also been investigated; these are listed in Table 10. 
Table 10. Performance of Other Supervised Learning Classification Methods for Brain Biometrics

\begin{tabular}{lllllll}
\hline Papers & \multicolumn{1}{c}{ Sbjs } & \multicolumn{1}{c}{ Chs } & \multicolumn{1}{c}{ Protocols } & \multicolumn{1}{c}{ Features } & \multicolumn{1}{c}{ Classifiers } & \multicolumn{1}{c}{ Performance } \\
\hline$[4]$ & 106 & 8 & $\begin{array}{l}\text { RC, RO, Motor, } \\
\text { Mental }\end{array}$ & PSD & GMM, UBM & HTER: $14.69 \%$ \\
{$[20]$} & 48 & 3 & RC & AR & Polynomial classifier & CRR: up to $96.08 \%$ \\
{$[50]$} & 4 & 16 & Mental & mAR & NBM & HTER: $1.5 \%-9.8 \%$ \\
{$[51]$} & 7 & 17 & Mental & AR & NBM & HTER: $0-12 \%$ \\
{$[66]$} & 40 & 4 & RC & AR, PSD & NBM & EER: $4.16 \%$ \\
{$[67]$} & 60 & 14 & ERP & WPD, RMS, std. & HMM & CRR: $97.5 \%$ \\
{$[75]$} & $20+30 I$ & 14 & Mental & DFT, mean, std. & HMM & FAR: $0-18.89 \%$ \\
{$[78]$} & 45 & $2,3,5$ & RC & AR & Polynomial classifier & CRR: up to $98.73 \%$ \\
{$[79]$} & 9 & 3,5 & RC, RO & AR & Linear classifier & CRR: up to $100 \%$ \\
{$[92]$} & 45 & 19 & RC, RO, Mental & AR, MFCC, & HMM & EER: up to $10 \%$ \\
& & & & bump modeling & & HTER: $0-52.1 \%$ \\
{$[100]$} & 9 & 8 & Mental & PSD & GMM, MAP & EER: $0.25 \%-16.99 \%$ \\
{$[112]$} & 9 & 3 & Mental & AR, PSD & RBF SVDD & EER: $0.13 \%-19.64 \%$ \\
& & & & GMM & DET curve
\end{tabular}

8.5.1 Gaussian Mixture Model (GMM). In the GMM, all feature vectors are assumed to be independent. Denoting that $\lambda$ is the parameter set for client $C$ and $\neg \lambda_{C}$ is the parameter set for a non-client, a claim for client $C$ 's identity is supported by the feature vectors $X, P\left(X \mid \lambda_{C}\right)$ is the likelihood of the claim made by the true client, and $P\left(X \mid \neg \lambda_{C}\right)$ is the likelihood of the claim coming from an impostor, then the authentication score $\Lambda(X)$ on the claim is shown in Equation (4). When the threshold is set to $\tau$, the claim is accepted when $\Lambda(X) \geq \tau$ and rejected otherwise $[4,100,112]$.

$$
\Lambda(X)=\log P\left(X \mid \lambda_{C}\right)-\log P\left(X \mid \neg \lambda_{C}\right) .
$$

8.5.2 Hidden Markov Model (HMM). HMM is used to process nonstationary signals and consists of three probability states: initial, transition, and observation, denoted by $\lambda=(\pi, A, B)$. For a model $\lambda$, an observation sequence $O=\left(O_{1}, O_{2}, \ldots, O_{T}\right)$ is modeled by GMM from a state sequence $Q=\left(Q_{1}, Q_{2}, \ldots, Q_{N}\right)$, then HMM seeks to find the best likelihood of $P(O \mid \lambda)[67,75,92]$.

8.5.3 Linear \& Polynomial Classifier. Denoting the feature vector as $x$, which is the observed feature vector of length $M$ in the linear terms of $x=\left[x_{1}, x_{2}, \ldots, x_{M}\right]^{T}$, and the transformation matrix as $G$, the linear classifier estimates the class labels $\hat{y}$ using a linear transformation so that $\hat{y}=x^{T} G$, where the transformation matrix $G$ is solved by minimizing the mean square error [79].

The polynomial classifier is similar to the linear classifier, except that it uses a polynomial format, quadratic terms, cubic terms, and so on, along with the linear terms of the feature vector. Instead of $x=\left[x_{1}, x_{2}, \ldots, x_{M}\right]^{T}$, the vector $z(x)=\left[1, x_{1}, x_{2}, \ldots, x_{M}, x_{1}^{2}, \ldots, x_{M}^{2}, x_{1} x_{2}\right.$, $\left.x_{1} x_{3}, \ldots, x_{1}^{3}, \ldots, x_{M}^{3}, \ldots, x_{1} x_{2} x_{3}, \ldots, x_{1}^{2} x_{2}, \ldots\right]$ is used. Then the polynomial classifier makes predictions using $\hat{y}=z^{T}(x) \cdot G[20,78]$. 
8.5.4 Naïve Bayes Model (NBM). NBM [137] is a probabilistic model based on the Bayes theorem with the assumption that features are strongly (naïve) independent. It has good performance when the dimension of the feature vectors is high. Assume that each element $x_{i}$ of the feature vector $X$ of dimension $M$ follows the Gaussian distribution and is statistically independent from each other; then the parameter $\theta$ of NBM can be estimated through Maximum Likelihood Estimation (MLE) using the training data. The likelihood of a claim is calculated as Equation (5). Given a threshold $\tau$, the authenticate claim will be accepted if $P(X \mid \theta) \geq \tau$, and otherwise rejected [50, 51, 66, 157].

$$
P(X \mid \theta)=\prod_{i=1}^{M} P\left(x_{i} \mid \theta_{i}\right) .
$$

8.5.5 Support Vector Data Description (SVDD). The key idea of SVDD [164] is to find a close boundary in which the targets are located. This is different from SVM, which aims to find a hyperplane to separate different classes. SVDD with an RBF kernel was adopted in several studies $[112,129,188]$. In order to reduce both the false acceptance rate and the false rejection rate simultaneously, Pham et al. [128] adopted the Small Sphere Two Large Margins Support Vector Data Description (SS2LM-SVDD), which constructed an optimal hypersphere in the feature space with one margin to include EEG the data of one person and with the remaining margins to exclude the EEG data belonging to other individuals.

\subsection{Unsupervised Feature Learning-Based Recognition}

So far, most of the methods used in brain biometric authentication and identification can be categorized into two types: similarity-based and supervised learning-based recognitions. Similaritybased methods measure the distances among the features for decision-making. However, the high noise level of the EEG signals could lower the accuracy of identifying individuals, and the computation efficiency decreases significantly as the sample size increases. For the supervised learningbased methods, features are extracted first from the EEG data before the supervised algorithms are adopted to train a model for prediction. Although supervised learning has shown its advantage in brain biometric recognition at fair computation times, its performance can be significantly affected by the quality of manually chosen features. If the feature represents the data well, a supervised learning algorithm can get good performance. Otherwise, the results will be unsatisfactory. Due to the complex nature of brain activities, EEG signals include a lot of noise, and random brain activities could influence the quality of the extracted features. Thus, unsupervised feature learning approaches that have achieved great success in the computer vision domain could be adopted in brain biometrics as well.

The Divergent Autoencoder (DIVA) [77] was used to reconstruct the ERPs automatically using an autoencoder, which is a three-layer network that has one input layer, one hidden layer, and one output layer; it tries to learn a function that directly maps the inputs to themselves. Based on this characteristic, autoencoder can reconstruct the input signal at the output. Then, DIVA was used to compare the similarity between the reconstructed input and stored templates during enrollment and made the decision. The identification accuracy was 54\% [144] and chance accuracy was $89 \%$ $[6,145]$.

A Convolutional Neural Network (CNN) [82], which is another method to learn intrinsic features automatically through the training process, has gained increasing attention recently due to its extraordinary capabilities of feature learning and classification and has also been explored for EEG-based user recognition. The convolution layers in CNN apply sets of filters to process small local parts of the input data, and these filters were replicated along the whole input space. Through a training process, features of the input data can be learned by these filters. Using "INPUT," 
"CONV," "AVGPOOL," "MAXPOOL," "FC," "DROPOUT," "RELU," "ELU,” "SOFTMAX," "mSVM," and "RF" to denote the possible layers (i.e., input, convolution, average pooling, max pooling, fully connected, dropout, relu, elu, softmax, multi-class SVM, and random forest, respectively), the network structure is presented in "[]" with "->" connecting adjacent layers, and a duplicate structure would be presented by the repeated number followed by the structure in "()". Chu et al. [27] investigated several deep learning models, including CNN, ANN, and RNN ending with the softmax, mSVM, and RF classifiers. The network architectures of CNN, ANN, and RNN were [INPUT $->3(\mathrm{CONV}->$ ELU $->$ MAXPOOL $->$ DROPOUT) $->3$ (FC $->$ ELU $->$ DROPOUT) $->$ SOFTMAX/mSVM/RF], [INPUT -> 4 (FC - > RELU -> DROPOUT) -> SOFTMAX/mSVM/RF], and [INPUT -> RNN -> FC -> SOFTMAX/mSVM/RF] correspondingly. Testing on 40 characteristics in high-risk individuals, 40 clinically stable first-episode patients with schizophrenia, and 40 healthy controls, respectively, using 64 channels under protocols of RC, RO, and watching video of news with neutral contents, the accuracy was $58.8 \%-83.9 \%, 62.2 \%-92.9 \%$, and $74.9 \%-99.2 \%$ for RNN, ANN, and CNN, respectively. The results showed that CNN performed best and RNN had the worse accuracy. Das et al. [33] used the CNN structure of [INPUT -> 2 (CONV -> MAXPOOL) -> CONV -> RELU -> FC -> SOFTMAX] to identify 40 subjects using 17-channel ERP data under the "geometric" protocol, and the CRR was in the range between $80.65 \%$ and $98.8 \%$. Gui et al. [46] proposed a one-dimensional CNN architecture to identify ERPs. From input to output, the CNN was composed of [INPUT -> CONV -> RELU -> MAXPOOL -> FC -> DROPOUT -> SOFTMAX]. Based on the dataset of 33 subjects of 30 channels using visual stimuli, the single-channel accuracy can reach between $99.6 \%$ and $100 \%$. Ma et al. [91] extracted spectrum-topographies under the resting state with eyes closed and open protocols as the 2-D inputs to the $\mathrm{CNN}$, which consisted of [INPUT $->2$ (CONV $->$ AVGPOOL) $->$ FC $->$ SOFTMAX], and obtained an accuracy of up to $88 \%$ in identifying 10 subjects. Mao et al. [98] used the CNN structure of [INPUT $->2$ (CONV -> RELU -> MAXPOOL) -> 2 (FC -> DROPOUT) - > FC - > SOFTMAX]. Testing on 100 subjects using 64 channels during a driving experiment, the CRR was 97\%. Schons et al. [152] used the CNN architecture of [INPUT -> 3 (CONV -> MAXPOOL) -> 4 (FC) - > DROPOUT -> SOFTMAX]. After finishing the training, the last three layers were removed and the output of the third FC layer was set as the features for verification. Testing on 109 subjects using 64 channels during RC and $\mathrm{RO}$, the EER was in the range of $0.19 \%$ to $11.2 \%$. Wu et al. [178] adopted the CNN structure of [INPUT -> 2 (CONV -> MAXPOOL) - > FC $->$ SOFTMAX] to authenticate 15 genuine users and 25 intruders using 16 channels using visual stimuli of self-face and non-self-face. The authentication accuracy was $92.4 \%$ with a FAR of $5.75 \%-6.71 \%$ and an FRR of $7.07 \%-8.49 \%$.

An attention-based encoder-decoder recurrent NN was implemented in Zhang et al. [186] to learn deep features for user identification. The feature learning modules included three components: the encoder formed by multiple fully connected layers and one recurrent Long Short-Term Memory (LSTM) layer, the attention module accepting the final hidden states and calculating better intermediate attention weights, and the decoder obtaining the learned deep features. Then, an extreme gradient boosting classifier, fused by a set of classification and regression trees, used the deep features learned from a delta band EEG for user identification. In testing on multitrial and single-trial datasets collected from 8 subjects and 14 channels under the RC protocol and the public EEGMMIDB database, the accuracies were $98.2 \%, 98.82 \%$, and $99.89 \%$ respectively.

\section{OPEN RESEARCH AREAS}

\subsection{Brain-Based Multimodal Biometrics}

Although the performance of brain biometrics is improving, it still suffers problems similar to other unimodal biometric systems, such as noisy data (e.g., eye blinks, muscle movements, or defects in 
Table 11. Performance of Brain Biometrics with Score- and Decision-Level Fusions

\begin{tabular}{|c|c|c|c|c|c|c|c|}
\hline \multirow[b]{2}{*}{ Papers } & \multirow[b]{2}{*}{ Sbjs } & \multirow[b]{2}{*}{ Chs } & \multirow[b]{2}{*}{ Protocols } & \multirow[b]{2}{*}{ Classifiers } & \multirow[b]{2}{*}{ Fusion Levels } & \multicolumn{2}{|c|}{ Performance } \\
\hline & & & & & & Before & After \\
\hline [6] & 30 & 1 & ERP & $\begin{array}{l}\text { CC, DIVA, } \\
\text { NDL, SVM }\end{array}$ & Decision (voting) & $\begin{array}{l}\text { Chance acc.: } \\
82 \%-92 \%\end{array}$ & $97 \%$ \\
\hline [7] & 5 & 14 & Mental & Linear SVM & Decision (voting) & CRR: $97.69 \%$ & $98.67 \%, 100 \%$ \\
\hline [40] & 109 & 64 & $\mathrm{RC}, \mathrm{RO}$ & $\begin{array}{l}\text { Euclidean } \\
\text { Mahalanobis }\end{array}$ & $\begin{array}{l}\text { Score (concatenation, } \\
\text { arithmetic mean, max, } \\
\text { min, geometric mean, } \\
\text { Bayes rule } \\
\text { likelihood ratio) }\end{array}$ & $\begin{array}{l}\text { EER: } 4.46 \%-10.6 \% \\
\text { FRR@1\%FAR: } \\
2.1 \%-3.5 \%\end{array}$ & $\begin{array}{l}1.42 \%-2.48 \% \\
1.76 \%-3.61 \%\end{array}$ \\
\hline [44] & 37 & 4 & ERP & Euclidean & Decision (SRBFNN) & CRR: $47.3 \%-67.57 \%$ & $82.7 \%-91.62 \%$ \\
\hline [80] & 36 & 3 & $\mathrm{RC}$ & LDA & $\begin{array}{l}\text { Score (multiplicative } \\
\text { inverse sum) }\end{array}$ & CRR: N/A & up to $99.69 \%$ \\
\hline [81] & 108 & 56 & $\mathrm{RC}, \mathrm{RO}$ & Mahalanobis & $\begin{array}{l}\text { Score (multiplicative } \\
\text { inverse sum) }\end{array}$ & CRR: up to $90.49 \%$ & up to $100 \%$ \\
\hline [97] & 50 & 19 & $\mathrm{RC}, \mathrm{RO}$ & $\begin{array}{l}\text { Manhattan } \\
\text { Euclidean } \\
\text { Cosine }\end{array}$ & $\begin{array}{l}\text { Score (mean) } \\
\text { Decision (voting) }\end{array}$ & CRR: N/A & up to $\sim 90 \%$ \\
\hline [130] & 25 & 19 & ERP & Manhattan & $\begin{array}{l}\text { Score (sum) } \\
\text { Decision (voting) }\end{array}$ & CRR: $66.93 \%-94.8 \%$ & $\begin{array}{l}88.27 \%-99.33 \% \\
97.33 \%-100 \%\end{array}$ \\
\hline [142] & 50 & 30 & ERP & $\mathrm{CC}$ & Decision (voting) & CRR: $60 \%-86 \%$ & $100 \%$ \\
\hline [147] & $50+20 \mathrm{I}$ & 2 & $\mathrm{RC}$ & $\begin{array}{l}\text { LDA, D-LDA, } \\
\text { QDA, D-QDA, } \\
\text { TREE, COP }\end{array}$ & $\begin{array}{l}\text { Score (mean, median, } \\
\text { OR) } \\
\text { Decision (voting) }\end{array}$ & EER: N/A & $\begin{array}{l}\text { mean: } 2.4 \% \\
\text { median: } 3 \% \\
\text { OR: } 4.8 \% \\
\text { voting: } 2.59 \%\end{array}$ \\
\hline
\end{tabular}

the capturing sensors), restricted degrees of freedom (e.g., feature similarities in systems with large user population), spoof attacks (e.g., in voice recognition, spoofing is done by recording the voice and using it to get access), and intra-class variations (e.g., user movements while being authenticated). To address those problems, multimodal biometric systems were proposed to eliminate the weakness of relying on one source of information in unimodal biometric systems [3, 15, 101, 141]. To integrate the information, fusion can be performed at the data or feature level, where the data itself or the extracted feature sets are fused; at the matching score level, where the matching scores generated by multiple classifiers are combined; or at the decision level, where the final output results of multiple classifiers are consolidated [141]. For brain biometrics, the most common fusion level is the feature level. Generally, multiple features are extracted from one or more channels and then concatenated together as a feature set for classification (as shown in Tables 6 to 10 in Section 8, which show two or more features or channels). Thus, in this section, only higher fusion levels (i.e., score and decision) are discussed. Table 11 summarizes the studies using a single EEG as a biometric trait but fusing at the score and decision levels. In score-level fusion, the scores could be integrated by operations like concatenation, arithmetic mean, maximum, minimum, geometric mean, Bayes rule, likelihood ratio, multiplicative inverse sum, sum, median, or OR [40, 80, 81, 97, $130,147]$. In decision-level fusion, the widely adopted fusion mechanism was voting [6, 7, 97, 130, 142,147 ]. Besides the voting method, Gui et al. [44] proposed the frequency-based and probabilitybased Simplified RBF Neural Network (SRBFNN) to combine the preliminary decision-level results to improve identification accuracy. The before and after fusion performance presented in the table shows that fusion techniques could improve EEG recognition performance.

Depending on the number of traits, sensors, and feature sets, multimodal biometrics can be classified into four scenarios: (i) single biometric trait, multiple sensors; (ii) single biometric trait, 
Table 12. Performance of Multiple Traits-Based Multimodal Biometric Systems

\begin{tabular}{|c|c|c|c|c|c|c|c|}
\hline \multirow[b]{2}{*}{ Papers } & \multirow[b]{2}{*}{ Sbjs } & \multirow[b]{2}{*}{ Chs } & \multirow[b]{2}{*}{ Protocols } & \multirow[b]{2}{*}{ Classifiers } & \multirow[b]{2}{*}{ Biometric Traits } & \multicolumn{2}{|c|}{ Performance } \\
\hline & & & & & & EEG & $\begin{array}{c}\text { Multiple } \\
\text { Traits }\end{array}$ \\
\hline [2] & 31 & 1 & $\begin{array}{l}\text { eye blink, } \\
\text { RC, ERP }\end{array}$ & LDA & EEG, eye blink & EER: $2.43 \%-4.4 \%$ & $0.89 \%-2.53 \%$ \\
\hline [9] & 9 & 61 & $\mathrm{RC}$ & Euclidean & EEG, ECG & EER: $11.17 \%$ & $2.94 \%$ \\
\hline [10] & 52 & 64 & $\begin{array}{l}\text { RC, RO, } \\
\text { Mental }\end{array}$ & $\begin{array}{l}\text { sum, product, } \\
\text { weighted } \\
\text { sum }\end{array}$ & EEG, ECG & EER: $22.97 \%-29.36 \%$ & $0.928 \%-8.216 \%$ \\
\hline [66] & 40 & 4 & $\mathrm{RC}$ & NBM & EEG, iris & EER: $4.16 \%$ & $2.12 \%$ \\
\hline [86] & 32 & 8 & ERP & DNN & EEG, EOG, GSR, Rb & CRR: $88 \%$ & $94 \%$ \\
\hline [103] & 32 & 32 & ERP & meta & EEG, EOG, GSR, Rb & CRR: $97.7 \%$ & $100 \%$ \\
\hline [118] & 5 & 19 & ERP & $\mathrm{NN}, \mathrm{LDA}$ & EEG, ECG & CRR: $76.9 \%-93.5 \%$ & $92.4 \%-99.8 \%$ \\
\hline [124] & 10 & 18 & Motor & Euclidean & EEG, hand kinematic & EER: N/A & $7.5 \%$ \\
\hline [135] & 14 & $\mathrm{~N} / \mathrm{A}$ & Mental & mean & EEG, face & CRR: $91.4 \%-93.8 \%$ & $100 \%$ \\
\hline [157] & 40 & 1 & $\mathrm{RC}$ & NBM & EEG, fingerprint & EER: $4.16 \%$ & $1.12 \%$ \\
\hline [172] & 6 & 19 & ERP & $\begin{array}{l}\text { sum, min, } \\
\max \end{array}$ & EEG, face & $\begin{array}{l}\text { FAR: } 0-20 \% \\
\text { GAR: } \sim 52 \%-91 \%\end{array}$ & $\begin{array}{l}0-20 \% \\
\sim 85 \%-100 \%\end{array}$ \\
\hline [178] & $15+25 I$ & 16 & ERP & least square & EEG, eye blink & $\begin{array}{l}\text { FAR: } 6.71 \% \\
\text { FRR: } 8.49 \%\end{array}$ & $\begin{array}{l}2.71 \% \\
2.09 \%\end{array}$ \\
\hline [185] & $6+2 \mathrm{I}$ & 64 & ERP & RNN, SVM & EEG, ID, Gait & $\begin{array}{l}\text { FAR: } 0 \\
\text { FRR: } 63.16 \%\end{array}$ & $\begin{array}{l}0 \\
1.9 \%\end{array}$ \\
\hline
\end{tabular}

multiple classifiers; (iii) single biometric trait, multiple units; and (iv) multiple biometric traits [141]. For brain biometrics, it is common to use multiple electrodes of the same type and that number into the hundreds, to collect EEG signals from different brain regions. Current studies have not considered the possibility of performance improvement by using different sensors for brain activity recordings. But multiple classifiers, multiple units, and multiple biometric traits have already been investigated. For example, Armstrong et al. [6] combined the results of CC, DIVA, NDL, and SVM and used multiple classifiers for the final decision-making. Sun [162] considered imagery tasks of moving left and right fingers separately; this use of multiple tasks data could help improve performance in correctly recognizing individuals compared to a single task. ECG and other types of biometric traits were also combined to seek better performance. Table 12 lists the papers which used multiple traits for authentication. EEG was combined with eye blinks $[2,178]$, ECG [9, 10, 118, 155], face [135, 172], iris [66], hand kinematic synergies [124], fingerprint [157], ID and gait [185], EOG, GSR, and a Respiration Belt (Rb) [86, 103], and performance improved.

\subsection{Security of Brain Biometrics}

Although brain biometrics have shown a high level of security in terms of signal acquisition and processing, they still share a similar identification/authentication architectural flow (i.e., sensors, feature extraction, a matching module, and a decision making module) with other biometrics such as fingerprint, face, and so on. Thus, brain biometric recognition systems are also vulnerable to modern cyber attacks. Currently, hill-climbing attacks to brain biometric recognition system were studied [93, 94], in which an attacker can access the matching scores produced by the matcher module and use them to generate synthetic EEG templates to successfully pass an authentication. The Bayesian algorithm, Nelder-Mead algorithm, Hooke-Jeeves algorithm, SPSA algorithm, and 
implicit algorithm were investigated and employed to address this risk. Gui et al. [45] studied the replay attack when the replayed ERPs were created by adding Gaussian noise to the original ERPs. Residual features varied between the ERPs with or without noise. An ensemble classifier combined with a low-level noise detector based on the ERPs' statistical variations was adopted to detect the replay attacks.

Moreover, an authorized person may be coerced to pass a recognition system. Su et al. [160] proposed an EEG-based identification system with a covert warning scheme. Besides thinking as instructed in protocols for EEG data collection, the users also generate three short bursts of muscle signals by clenching their teeth to code covert warning messages along with the EEG signals. Even though the input EEG identity might pass the recognition system, the input user would still be considered as unauthorized if the covert warning message was detected.

A popular approach to securing biometric authentication systems is to establish cancelable biometric templates [125], for example through various types of revocable and noninvertible template transformations. Lin et al. [88] argued that cancelability can be achieved for brain-based biometrics by nature, in that the manipulation of visual stimulus can elicit a new ERP biometric template while preserving its intrinsic uniqueness. In other words, the change in visual stimulation can alter the ERP signal of individuals and allow a truly cancelable biometric.

\subsection{Permanence of Brain Biometrics}

Permanence, which represents the intra-individual stability of brain activities over a period of time, is an important characteristic and should be carefully investigated to provide sufficient evidence to support brain biometrics. Although many studies have worked on the reproducibility of intra- and inter-individual psychophysiological features [19], it is only recently that researchers examined reproducibility in the area of brain biometrics. These experiments asked participants to return to the lab for more data recording sessions after a certain amount of time following their first visit. The identification accuracy of different sessions was analyzed by setting the first session as a performance reference. Table 13 lists studies which investigated the permanence of brain biometrics. Generally, most of the permanence studies recorded two [33, 79, 84, 96, 106, 130, 143, 174, 177, 178] or three sessions [6, 32, 34, 36, 54, 97, 146]. Maiorana and Campisi [92] extended the experiment to 6 sessions. The time durations among the sessions varied from about 1 week [6, $32-34,79,92,97,106,146,174]$ to approximately 36 [92] months from the first EEG data recording date. With data from one or more sessions as a training set and data from all the sessions or other different sessions as a testing set, typically, the identification or authentication performance was maintained or degraded only slightly. These results demonstrated that EEG can be a biometric that can last for a long time.

\subsection{Stability of Brain Biometrics}

EEG is known to be sensitive to emotional and mental states. Therefore, Pham et al. [129] studied the stability of EEG biometrics on a publicly available emotional EEG database, DEAP. During the enrollment phase, only data of calm emotional state was selected. But for the verification phase, emotional states of calm, stress, and excited were used. The results showed that the calm state had better EER than the stress and excited states when the data length was about 1-1.5s, suggesting a good choice for anticoercion attacks. As the length increased, stress gave better performance. When the data length reached $8 \mathrm{~s}$, the performance of the three emotions was very close. Thus this could be an optimal data length to tolerate emotion effects in EEG-based biometric authentication.

Moreover, diet is also thought to have an impact on EEG signals. There are studies investigating the EEG signals in the resting protocols with an intake of pure water and coffee $[66,160]$, but 
Table 13. Permanence of Brain Biometrics

\begin{tabular}{|c|c|c|c|c|c|c|c|c|c|}
\hline \multirow[b]{2}{*}{ Papers } & \multirow[b]{2}{*}{ Sbjs } & \multirow[b]{2}{*}{ Chs } & \multirow[b]{2}{*}{ Protocols } & \multirow[b]{2}{*}{ Sessions } & \multirow[b]{2}{*}{ Features } & \multirow[b]{2}{*}{ Classifiers } & \multicolumn{3}{|c|}{ Performance } \\
\hline & & & & & & & 1st & 2nd & 3rd \\
\hline [6] & 30 & 1 & ERP & $\begin{array}{l}1 \text { ( } 30 \text { sbjs }) \\
2 \text { (15 sbjs, } 5-40 \text { days }) \\
3 \text { ( } 9 \text { sbjs, } 134-188 \text { days })\end{array}$ & raw ERP & $\begin{array}{l}\text { CC, DIVA, } \\
\text { NDL, SVM, } \\
\text { Meta }\end{array}$ & $\begin{array}{l}\text { Chance acc.: } \\
82 \%-97 \%\end{array}$ & $89 \%$ & $93 \%$ \\
\hline [32] & 50 & 6,17 & ERP & $\begin{array}{l}2 \text { (1 week) } \\
3 \text { (25-49 days) }\end{array}$ & raw ERP & Cosine & EER: N/A & $\sim 8 \%-18 \%$ & $\sim 10 \%-25 \%$ \\
\hline [33] & 40 & 17 & ERP & 2 (1 week) & raw ERP & $\mathrm{CNN}$ & CRR: N/A & $80.65 \%-98.8 \%$ & \\
\hline [34] & 50 & 6,17 & ERP & $\begin{array}{l}2 \text { (1 week) } \\
3 \text { (25-49 days) }\end{array}$ & raw ERP & DTW & EER: N/A & $\sim 7 \%-17 \%$ & $\sim 10 \%-25 \%$ \\
\hline [36] & 8 & 32 & ERP & $\begin{array}{l}2 \text { ( } 3 \text { weeks) } \\
3 \text { (6 weeks) }\end{array}$ & variance & $\mathrm{kNN}$ & $\begin{array}{l}\text { GAR: N/A } \\
\text { FAR: N/A }\end{array}$ & $\begin{array}{l}\text { GAR: } 92.5 \% \\
\text { FAR: } 0.9 \%\end{array}$ & $\begin{array}{l}\text { GAR: } 91.3 \% \\
\text { FAR: } 1 \%\end{array}$ \\
\hline [54] & 50 & 19 & RO & 3 (1 month) & $\mathrm{AR}$ & Manhattan & CRR: N/A & up to $95 \%$ & \\
\hline [79] & 9 & 3,5 & $\mathrm{RC}, \mathrm{RO}$ & 2 (1-3 weeks) & AR & Linear & $\begin{array}{l}\text { CRR: } \\
91.8 \%-100 \%\end{array}$ & $43.46 \%-100 \%$ & \\
\hline [84] & 4 & 1 & $\mathrm{RC}$ & 2 (10 days- 5 months) & $\begin{array}{l}\alpha \text { frequency } \\
\text { MP, SP }\end{array}$ & LDA & CRR: N/A & $86.7 \%-100 \%$ & \\
\hline [92] & 45 & 19 & $\begin{array}{l}\mathrm{RC}, \mathrm{RO} \text {, } \\
\text { Mental }\end{array}$ & $\begin{array}{l}2 \text { ( 1 week) } \\
3 \text { ( 1 month) } \\
4 \text { ( 7 months) } \\
5 \text { ( 16 months) } \\
6 \text { (30 sbjs, } \sim 36 \text { months })\end{array}$ & $\begin{array}{l}\text { AR, MFCC, } \\
\text { Bump modeling }\end{array}$ & HMM & $\begin{array}{l}\text { EER: } \\
\sim 2 \%-6 \%\end{array}$ & $\sim 1 \%-8 \%$ & $\begin{array}{l}\sim 2 \%-10 \% \text { (3rd) } \\
\sim 4 \%-10 \% \text { (4th) } \\
\sim 3 \%-11 \% \text { (5th) }\end{array}$ \\
\hline [96] & 30 & 19 & $\mathrm{RC}, \mathrm{RO}$ & 2 (1 month) & $\begin{array}{l}\text { EB, ETB, } \\
\text { LDA, PSD }\end{array}$ & $\begin{array}{l}\text { Cosine, } \\
\text { Euclidean, } \\
\text { Manhattan, } \\
\text { Mahalanobis }\end{array}$ & CRR: N/A & up to $90 \%$ & \\
\hline [97] & 50 & 19 & $\mathrm{RC}, \mathrm{RO}$ & $\begin{array}{l}2(\sim 1 \text { week }) \\
3(\sim 34 \text { days })\end{array}$ & $\mathrm{AR}, \mathrm{COH}, \mathrm{PSD}$ & $\begin{array}{l}\text { Manhattan } \\
\text { Euclidean } \\
\text { Cosine }\end{array}$ & CRR: N/A & up to $\sim 85 \%$ & up to $\sim 86 \%$ \\
\hline [100] & 9 & 8 & Mental & 12 (3 days) & PSD & GMM, MAP & $\begin{array}{l}\text { HTER: } \\
7.1 \%-16.1 \%\end{array}$ & $34.9 \%-45.5 \%$ & $36.2 \%-51.5 \%$ \\
\hline [106] & $15+5 I$ & 2 & $\mathrm{RC}$ & 2 (5-15 days) & AR, PSD & LDA & $\begin{array}{l}\text { CRR: N/A } \\
\text { GAR: N/A } \\
\text { HTER: N/A }\end{array}$ & $\begin{array}{l}67.8 \% \\
95.5 \% \\
18.2 \%\end{array}$ & \\
\hline [130] & 25 & 19 & ERP & 2 (15 days) & AR, MFCC & Manhattan & CRR: N/A & $66.93 \%-94.8 \%$ & \\
\hline [143] & 20 & 26 & ERP & 2 (48-516 days) & raw ERP & $\mathrm{CC}$ & CRR: $100 \%$ & $100 \%$ & \\
\hline [146] & 30 & 1 & ERP & $\begin{array}{l}1 \text { ( } 30 \text { sbjs }) \\
2 \text { (15 sbjs, } 5-40 \text { days }) \\
3 \text { ( } 9 \text { sbjs, } 134-188 \text { days })\end{array}$ & raw ERP & $\mathrm{CC}$ & CRR: $99 \%$ & $93 \%$ & $84 \%$ \\
\hline [174] & 4 & 128 & $\mathrm{RC}$ & 2 (1 week) & CWT & Euclidean & $\begin{array}{l}\text { CRR: } \\
78.16 \%-99.73 \%\end{array}$ & $51.09 \%-92.58 \%$ & \\
\hline [177] & $15+30 \mathrm{I}$ & 16 & ERP & 2 ( $\sim 30$ days $)$ & $\begin{array}{l}\text { weighted } \\
\text { average } \\
\text { logistic } \\
\text { regression }\end{array}$ & HDCA & FRR: $7.85 \%$ & $7.27 \%$ & \\
\hline [178] & $15+25 I$ & 16 & ERP & 2 ( $\sim 30$ days $)$ & raw ERP & $\mathrm{CNN}$ & FRR: $8.49 \%$ & $7.07 \%$ & \\
\hline
\end{tabular}

there was no comparison result reported. In Su et al. [158], the authors analyzed circadian and diet effects. Forty subjects participated in experiments with pure water for the first period and the same amount of coffee for the second period. In each period, there were 6 sessions. After one pre-dose session that started at 9:00 am, the subjects drank water or coffee. Then five post-dose sessions were recorded every 0.5 hour after drinking (i.e., $0.5-2.5$ hours). The results showed that factors like diet and time of the day could diminish the identification accuracy by almost $30 \%$. 


\section{CONCLUSION}

In this article, we presented a survey of brain biometrics, which possess some unique characteristics and advantages over conventional biometrics and thus have gained increasing attention in the community. We surveyed the recent literature and reviewed some background on the acquisition, processing, and analysis of brain signals. For data acquisition, we reviewed commercially available EEG devices, recording protocols, and publicly available databases for brain biometrics. To analyze the EEG data, we presented popular pre- and postprocessing methods, frequently used features and feature/channel selection techniques, dimensionality reduction methods, and classifiers. In addition, we also discussed and highlighted some of the open research areas in the field, including multimodal biometrics which have EEG as one of the biometric traits, and the security, permanence, and stability of brain biometrics. Finally, we summarized a table of terminology and abbreviations (see Table 1 of the Supplementary Online-only Material) that have been introduced and used in the literature.

Above and beyond the practical considerations we have presented, we argue that brain biometrics have theoretical advantages over other biometric credentials. Brain biometrics in general cannot be collected without a user's knowledge and consent, which makes them resistant to theft. In particular, ERP-based brain biometrics are nonvolitional, meaning that users are neither aware of nor in control of these responses. This makes them resistant to disclosure. Brain biometrics also are resistant to coercion as brain activity is altered by events that cause stress. Brain biometrics are cancelable in that they can be acquired from numerous distinct functional brain systems (e.g., the memory system, the decision-making system, the facial recognition system). The theoretical motivation for exploring brain biometrics is thus strong, and the emerging literature that we have reviewed here demonstrates that their theoretical advantages are accompanied by empirically demonstrated feasibility.

\section{REFERENCES}

[1] M. K. Abdullah, K. S. Subari, J. L. C. Loong, and N. N. Ahmad. 2010. Analysis of effective channel placement for an EEG-based biometric system. In Proceedings of the 2010 IEEE EMBS Conference on Biomedical Engineering and Science (IECBES'10). IEEE, 303-306.

[2] M. Abo-Zahhad, S. M. Ahmed, and S. N. Abbas. 2016. A new multi-level approach to EEG based human authentication using eye blinking. Pattern Recognition Letters 82 (2016), 216-225.

[3] O. Al-Hamdani, A. Chekima, J. Dargham, S.-H. Salleh, F. Noman, H. Hussain, A. K. Ariff, and A. M. Noor. 2013. Multimodal biometrics based on identification and verification system. fournal of Biometrics Biostatistics 4, 2 (Mar. 2013), 1-8.

[4] S. Altahat, M. Wagner, and E. M. Marroquin. 2015. Robust electroencephalogram channel set for person authentication. In Proceedings of the 2015 IEEE International Conference on Acoustics, Speech \& Signal Processing (ICASSP'15). IEEE, 997-1001.

[5] C. W. Anderson, E. A. Stolz, and S. Shamsunder. 1998. Multivariate autoregressive models for classification of spontaneous electroencephalographic signals during mental tasks. IEEE Transactions in Biomedical Engineering 45, 3 (1998), 277-286.

[6] B. C. Armstrong, M. V. Ruiz-Blondet, N. Khalifian, K. J. Kurtz, Z. Jin, and S. Laszlo. 2015. Brainprint: Assessing the uniqueness, collectability, and permanence of a novel method for ERP biometrics. Neurocomputing 166 (2015), 59-67.

[7] C. Ashby, A. Bhatia, F. Tenore, and J. Vogelstein. 2011. Low-cost electroencephalogram (EEG) based authentication. In Proceedings of the 5th International IEEE/EMBS Conference on Neural Engineering (NER'11). IEEE, 442-445.

[8] Y. Bai, Z. Zhang, and D. Ming. 2014. Feature selection and channel optimization for biometric identification based on visual evoked potentials. In Proceedings of the 19th International Conference on Digital Signal Processing (DSP'14). IEEE, 772-776.

[9] S. Barra, A. Casanova, M. Fraschini, and M. Nappi. 2015. EEG/ECG signal fusion aimed at biometric recognition. In Proceedings of the International Conference on Image Analysis \& Processing (ICIAP'15). Springer, 35-42.

[10] S. Barra, A. Casanova, M. Fraschini, and M. Nappi. 2017. Fusion of physiological measures for multimodal biometric systems. Multimedia Tools and Applications 76, 4 (2017), 4835-4847. 
[11] M. K. Bashar, I. Chiaki, and H. Yoshida. 2016. Human identification from brain EEG signals using advanced machine learning method EEG-based biometrics. In Proceedings of the 2016 IEEE EMBS Conference on Biomedical Engineering \& Science (IECBES'16). IEEE, 475-479.

[12] L. Bi, X.-A. Fan, and Y. Liu. 2013. EEG-based brain-controlled mobile robots: A survey. IEEE Transactions on HumanMachince Systems 43, 2 (2013), 161-176.

[13] B. Blankertz, K. R. Müller, G. Curio, T. M. Vaughan, G. Schalk, J. R. Wolpaw, A. Schlögl, C. Neuper, G. Pfurtscheller, T. Hinterberger, M. Schröder, and N. Birbaumer. 2004. The BCI competition 2003: Progress and perspectives in detection and discrimination of EEG single trials. IEEE Transactions on Biomedical Engineering 51, 6 (June 2004), 1044-1051.

[14] B. Blankertz, K. R. Müller, D. J. Krusienski, G. Schalk, J. R. Wolpaw, A. Schlögl, G. Pfurtscheller, J. del R. Millán, M. Schröder, and N. Birbaumer. 2006. The BCI competition III: Validating alternative approaches to actual BCI problems. IEEE Transactions on Neural Systems Rehabilitation Engineering 14, 2 (June 2006), 153-159.

[15] K. W. Bowyer, K. I. Chang, P. Yan, P. J. Flynn, E. Hansley, and S. Sarkar. 2006. Multi-modal biometrics: An overview In Proceedings of the 2nd Workshop Multi-Modal User Authentication. 1-8. http://mmua.cs.ucsb.edu/.

[16] K. Brigham and B. V. K. V. Kumar. 2010. Subject identification from electroencephalogram (EEG) signals during imagined speech. In Proceedings of the 4th IEEE International Conference on Biometrics: Theory Application \& Systems (BTAS'10). IEEE, 1-8.

[17] C. Brunner, R. Leeb, G. R. Müller-Putz, A. Schlögl, and G. Pfurtscheller. 2008. BCI Competition 2008-Graz data set A. Institute for Knowledge Discovery (Laboratory of Brain-Computer Interfaces), Graz University of Technology (2008), 16.

[18] D. Cai, K. Liu, and F. Su. 2015. Local metric learning for EEG-based personal identification. In Proceedings of the IEEE International Conference on Acoustics, Speech \& Signal Processing (ICASSP’15). IEEE, 842-846.

[19] P. Campisi and D. La Rocca. 2014. Brain waves for automatic biometric-based user recognition. IEEE Transactions on Information and Forensic Security 9, 5 (May 2014), 782-800.

[20] P. Campisi, G. Scarano, F. Babiloni, F. DeVico Fallani, S. Colonnese, E. Maiorana, and L. Forastiere. 2011. Brain waves based user recognition using the "eyes closed resting conditions" protocol. In Proceedings of the 2011 IEEE International Workshop on Information Forensics \& Security (WIFS'11). IEEE, 1-6.

[21] K. Cao and A. K. Jain. 2016. Hacking Mobile Phones Using 2D Printed Fingerprints. Technical Report MSU-CSE-16-2. Michigan State University.

[22] J. F. Cavanagh and J. J. B. Allen. 2008. Multiple aspects of the stress response under social evaluative threat: An electrophysiological investigation. Psychoneuroendocrinology 33, 1 (2008), 41-53.

[23] Chaos Computer Club (CCC). 2014. Fingerprint Biometrics hacked again. https://www.ccc.de/en/updates/2014/ursel.

[24] Y. Chen, A. D. Atnafu, I. Schlattner, W. T. Weldtsadik, M.-C. Roh, H. J. Kim, S.-W. Lee, B. Blankertz, and S. Fazli. 2016. A high-security EEG-based login system with RSVP stimuli and dry electrodes. IEEE Transactions on Information Forensics and Security 11, 12 (2016), 2635-2647.

[25] Y. M. Chi, T.-P. Jung, and G. Cauwenberghs. 2010. Dry-contact and noncontact biopotential electrodes: Methodological review. IEEE Review of Biomedical Engineering 3 (2010), 106-119.

[26] Y. M. Chi, Y.-T. Wang, Y. Wang, C. Maier, T.-P. Jung, and G. Cauwenberghs. 2011. Dry and noncontact EEG sensors for mobile brain computer interfaces. IEEE Transactions on Neural System Rehabilitation Engineering 20, 2 (Dec. 2011), $228-235$

[27] L. Chu, R. Qiu, H. Liu, Z. Ling, and X. Shi. 2017. Individual recognition in schizophrenia using deep learning methods with random forest and voting classifiers: Insights from resting state EEG streams. arXiv Preprint arXiv:1707.03467 (2017), $1-7$.

[28] J. Chuang, H. Nguyen, C. Wang, and B. Johnson. 2013. I think, therefore I am: Usability and security of authentication using brainwaves. In Financial Cryptography and Data Security, Andrew A. Adams, Michael Brenner, and Smith Matthew (Eds.). Springer, 1-16. https://www.springer.com/us/book/9783642413193.

[29] A. Crobe, M. Demuru, L. Didaci, G. L. Marcialis, and M. Fraschini. 2016. Minimum spanning tree and k-core decomposition as measure of subject-specific EEG traits. Biomed. Phys. Eng. Expr. 2, 1 (2016), 017001.

[30] Y. Dai, X. Wang, X. Li, and Y. Tan. 2015. Sparse EEG compressive sensing for web-enabled person identification. Measurement 74 (2015), 11-20.

[31] K. Das, S. Zhang, B. Giesbrecht, and M. P. Eckstein. 2009. Using rapid visually evoked EEG activity for person identification. In Proceedings of the 2009 Annual International Conference on IEEE Engineering in Medicine \& Biology Society (EMBC'09). IEEE, 2490-2493.

[32] R. Das, E. Maiorana, and P. Campisi. 2016. EEG biometrics using visual stimuli: A longitudinal study. IEEE Signal Processing Letters 23, 3 (2016), 341-345.

[33] R. Das, E. Maiorana, and P. Campisi. 2017. Visually evoked potential for EEG biometrics using convolutional neural network. In Proceedings of the 25th European Signal Processing Conference (EUSIPCO'17). IEEE, 951-955. 
[34] R. Das, E. Piciucco, E. Maiorana, and P. Campisi. 2016. Visually evoked potentials for EEG biometric recognition. In Proceedings of the 1st International Workshop on Sensors, Processing \& Learning for Intelligent Machinces (SPLINE'16). IEEE, $1-5$.

[35] P. Davis, C. D. Creusere, and J. Kroger. 2014. Classification of human viewers using high-resolution EEG with SVM. In Proceedings of the 48th Asilomar Conference on Signals, Systems \& Computing (ACSSC'14). 184-188.

[36] O. Falzon, R. Zerafa, T. Camilleri, and K. P. Camilleri. 2017. EEG-based biometry using steady state visual evoked potentials. In Proceedings of the 39th Annual International Conference on IEEE Engineering in Medicine \& Biology Soc. (EMBC'17). IEEE, 4159-4162.

[37] A. Ferreira, C. Almeida, P. Georgieva, A. Tomé, and F. Silva. 2010. Advances in EEG-Based biometry. In Image Analysis and Recognition (ICIAR'10), A. Campilho and M. Kamel (Eds.). Lecture Notes in Computer Science, Vol. 6112. Springer, Berlin, Heidelberg. https://link.springer.com/chapter/10.1007/978-3-642-13775-4_29\#citeas.

[38] K. Fladby. 2008. Brain Wave Based Authentication. Master's thesis. Gj申vik University College.

[39] M. Fraschini, A. Hillebrand, M. Demuru, L. Didaci, and G. L. Marcialis. 2015. An EEG-based biometric system using eigenvector centrality in resting state brain networks. IEEE Signal Processing Letters 22, 6 (2015), 666-670.

[40] M. Garau, M. Fraschini, L. Didaci, and G. L. Marcialis. 2016. Experimental results on multi-modal fusion of EEGbased personal verification algorithms. In Proceedings of the 2016 International Conference on Biometrics (ICB'16). IEEE, $1-6$.

[41] A. L. Goldberger, L. A. Amaral, L. Glass, J. M. Hausdorff, P. C. Ivanov, R. G. Mark, J. E. Mietus, G. B. Moody, C. K. Peng, and H. E. Stanley. 2000. Physiobank, physiotoolkit, and physionet components of a new research resource for complex physiologic signals. Circulation 101, 23 (2000), e215-e220.

[42] Q. Gui, Z. Jin, M. V. Ruiz-Blondet, S. Laszlo, and W. Xu. 2015. Towards EEG biometrics: Pattern matching approaches for user identification. In Proceedings of the 2015 IEEE International Conference on Identity, Security, and Behavioral Analysis (ISBA'15). IEEE, 1-6.

[43] Q. Gui, Z. Jin, and W. Xu. 2014. Exploring EEG-based biometrics for user identification and authentication. In Proceedings of the 2014 IEEE Signal Processing in Medicine \& Biology Symposium (SPMB'14). IEEE, 1-6.

[44] Q. Gui, Z. Jin, W. Xu, M. V. Ruiz-Blondet, and S. Laszlo. 2015. Multichannel EEG-based biometric using improved RBF neural networks. In Proceedings of the 2015 IEEE Signal Processing in Medicine \& Biology Symposium (SPMB'15). IEEE, 1-6.

[45] Q. Gui, W. Yang, Z. Jin, M. V. Ruiz-Blondet, and S. Laszlo. 2016. A residual feature-based replay attack detection approach for brainprint biometric systems. In Proceedings of the 8th IEEE International Workshop on Information, Forensics \& Security (WIFS'16). IEEE, 1-6.

[46] Q. Gui, W. Yang, M. V. Ruiz-Blondet, S. Laszlo, and Z. Jin. 2016. An unsupervised, EEG-based person identification approach using convolutional neural networks. In Proceedings of the 42nd Annual Northeast Bioengineering Conference (NEBEC'16). 1-2.

[47] I. Guyon, J. Weston, S. Barnhill, and V. Vapnik. 2002. Gene selection for cancer classification using support vector machines. Machine Learning 46, 1-3 (2002), 389-422.

[48] C. J. Harland, T. D. Clark, and R. J. Prance. 2002. Remote detection of human electroencephalograms using ultrahigh input impedance electric potential sensors. Applied Physics Letters 81, 17 (2002), 3284-3286.

[49] R. S. Harshit, K. P. Thomas, K. G. Smitha, and A. P. Vinod. 2016. Online electroencephalogram (EEG) based biometric authentication using visual and audio stimuli. In Proceedings of the 2016 IEEE EMBS Conference on Biomedical Engineering \& Science (IECBES'16). IEEE, 454-459.

[50] C. He, X. Lv, and Z. J. Wang. 2009. Hashing the mAR coefficients from EEG data for person authentication. In Proceedings of the 2009 IEEE Intenational Conference on Acoustics, Speech \& Signal Processing (ICASSP'09). 1445-1448.

[51] C. He and J. Wang. 2009. An independent component analysis (ICA) based approach for EEG person authentication. In Proceedings of the 3rd International Conference on Bioinformatics and Biomedical Engineering (ICBBE'09). 1-4.

[52] C. R. Hema and A. A. Osman. 2010. Single trial analysis on EEG signatures to identify individuals. In Proceedings of the 6th International Colloquoy on Signal Processing \& Its Applications (CSPA'10). IEEE, 1-3.

[53] C. R. Hema, M. P. Paulraj, and H. Kaur. 2008. Brain signatures: A modality for biometric authentication. In Proceedings of the 2008 International Conference on Electronic Design (ICED'08). 1-4.

[54] G. E. Hine, E. Maiorana, and P. Campisi. 2017. Resting-state EEG: A study on its non-stationarity for biometric applications. In Proceedings of the 2017 International Conference on Biometrics Special Interest Group (BIOSIG'17). $1-5$.

[55] X. Huang, S. Altahat, D. Tran, and D. Sharma. 2012. Human identification with electroencephalogram (EEG) signal processing. In Proceedings of the 2012 International Symposium on Communication \& Information Technology (ISCIT'12). IEEE, 1021-1026.

[56] H. J. Hwang, S. Kim, S. Choi, and C. H. Im. 2013. EEG-based brain-computer interfaces: A thorough literature survey. International fournal on Human-Computer Interactions 29, 12 (2013), 814-826. 
[57] ISO/IEC 2382-37:2017(en) 2017. Information Technology - Vocabulary - Part 37: Biometrics. Standard. International Organization for Standardization (ISO).

[58] A. Jain, R. Bolle, and S. Pankanti. 2006. Biometrics: Personal Identification in Networked Society. Vol. 479. Springer Science \& Business Media.

[59] A. K. Jain, A. Ross, and S. Prabhakar. 2004. An introduction to biometric recognition. IEEE Transactions on Circuits Systems and Video Technology 14, 1 (2004), 4-20.

[60] A. K. Jaiswal and H. Banka. 2017. Brain signal based biometric identification using one-dimensional local gradient pattern and artificial neural network. In Proceedings of the International Conference on Computer Intelligence, Communications, \& Business Analytics (CICBA'17). Springer, 525-536.

[61] I. Jayarathne, M. Cohen, and S. Amarakeerthi. 2016. BrainID: Development of an EEG-based biometric authentication system. In Proceedings of the IEEE 7th Annual Information Technology, Electronics \& Mobile Communication Conference (IEMCON'16). IEEE, 1-6.

[62] B. Johnson, T. Maillart, and J. Chuang. 2014. My thoughts are not your thoughts. In Proceedings of the 2014 ACM International foint Conference on Pervasive \& Ubiquitous Computing: Adjunct Publication (UbiComp'14 Adjunct). ACM, 1329-1338.

[63] C. A. Joyce, I. F. Gorodnitsky, and M. Kutas. 2004. Automatic removal of eye movement and blink artifacts from EEG data using blind component separation. Psychophysiology 41, 2 (Mar. 2004), 313-325.

[64] J.-H. Kang, Y. C. Jo, and S.-P. Kim. 2018. Electroencephalographic feature evaluation for improving personal authentication performance. Neurocomputing 287 (2018), 93-101.

[65] J.-H. Kang, C. H. Lee, and S.-P. Kim. 2016. EEG feature selection and the use of Lyapunov exponents for EEGbased biometrics. In Proceedings of the 2016 IEEE EMBS International Conference on Biomedical \& Health Informatics (BHI'16). IEEE, 228-231.

[66] T. Kathikeyan and B. Sabarigiri. 2012. Countermeasures against iris spoofing and liveness detection using electroencephalogram (EEG). In Proceedings of the 2012 International Conference on Computing, Communications \& Applications (ICCCA'12). IEEE, 1-5.

[67] B. Kaur, D. Singh, and P. P. Roy. 2017. A novel framework of EEG-based user identification by analyzing musiclistening behavior. Multimedia Tools and Applications 76, 24 (2017), 25581-25602.

[68] Z. A Keirn and J. I. Aunon. 1990. A new mode of communication between man and his surroundings. IEEE Transactions on Biomedical Engineering 37, 12 (1990), 1209-1214.

[69] S. Keshishzadeh, A. Fallah, and S. Rashidi. 2016. Improved EEG based human authentication system on large dataset In Proceedings of the 24th Iran Conference on Electrical Engineering (ICEE'16). IEEE, 1165-1169.

[70] W. Khalifa, A. Salem, M. Roushdy, and K. Revett. 2012. A survey of EEG based user authentication schemes. In Proceedings of the 8th International Conference on Information \& Systems (INFOS'12). BIO-55-BIO-60.

[71] J. Klonovs, C. K. Petersen, H. Olesen, and A. Hammershoj. 2013. ID proof on the go: Development of a mobile EEG-based biometric authentication system. IEEE Vehicular Technology Magazine 8, 1 (Mar. 2013), 81-89.

[72] S. Koelstra, C. Muhl, M. Soleymani, J. S. Lee, A. Yazdani, T. Ebrahimi, T. Pun, A. Nijholt, and I. Patras. 2012. DEAP: A database for emotion analysis using physiological signals. IEEE Transactions on Affective Computing 3, 1 (Jan. 2012), 18-31.

[73] T. Koike-Akino, R. Mahajan, T. K. Marks, Y. Wang, S. Watanabe, O. Tuzel, and P. Orlik. 2016. High-accuracy user identification using EEG biometrics. In Proceedings of the 38th Annual International Conference of the IEEE Engineering in Medicince \& Biology Society (EMBC’16). IEEE, 854-858.

[74] W. Kong, Q. Fan, L. Wang, B. Jiang, Y. Peng, and Y. Zhang. 2017. Task-free brainprint recognition based on degree of brain networks. In Proceedings of the International Conference on Neural Information Processing (ICONIP'17). Springer, 709-717.

[75] P. Kumar, R. Saini, P. P. Roy, and D. P. Dogra. 2017. A bio-signal based framework to secure mobile devices. fournal of Network Computing Applications 89 (2017), 62-71.

[76] P. Kumari and A. Vaish. 2015. Brainwave based user identification system: A pilot study in robotics environment. Robotics and Autonomic Systems 65 (2015), 15-23.

[77] K. J. Kurtz. 2007. The divergent autoencoder (DIVA) model of category learning. Psychonomic Bulletin Reviews 14, 4 (2007), 560-576.

[78] D. La Rocca, P. Campisi, and G. Scarano. 2012. EEG biometrics for individual recognition in resting state with closed eyes. In Proceedings of the 2012 International Conference on Biometrics Specical Interest Group (BIOSIG'12). 1-12.

[79] D. La Rocca, P. Campisi, and G. Scarano. 2013. On the repeatability of EEG features in a biometric recognition framework using a resting state protocol. In BIOSIGNALS. 419-428.

[80] D. La Rocca, P. Campisi, and J. Solé-Casals. 2013. EEG based user recognition using BUMP modelling. In Proceedings of the 2013 International Conference on Biometrics Special Interest Group (BIOSIG'13). 1-12. 
[81] D. La Rocca, P. Campisi, B. Vegso, P. Cserti, G. Kozmann, F. Babiloni, and F. D. V. Fallani. 2014. Human brain distinctiveness based on EEG spectral coherence connectivity. IEEE Transactions on Biomedical Engineering 61, 9 (Sept. 2014), 2406-2412.

[82] Y. LeCun, L. Bottou, Y. Bengio, and P. Haffner. 1998. Gradient-based learning applied to document recognition. Proceedings of the IEEE 86, 11 (Nov. 1998), 2278-2324.

[83] C. Lee, J.-H. Kang, and S.-P. Kim. 2016. Feature slection using mutual information for EEG-based biometrics. In Proceedings of the 39th International Conference on Telecommunications \& Signal Processing (TSP'16). IEEE, 673-676.

[84] H. J. Lee, H. S. Kim, and K. S. Park. 2013. A study on the reproducibility of biometric authentication based on electroencephalogram (EEG). In Proceedings of the 6th International IEEE/EMBS Conference on Neural Engineering (NER'13). 13-16.

[85] R. Leeb, C. Brunner, G. R. Müller-Putz, A. Schlögl, and G. Pfurtscheller. 2008. BCI Competition 2008-Graz data set B. Graz University of Technology, Austria (2008), 1-6.

[86] Y. Li, Y. Zhao, T. Tan, N. Liu, and Y. Fang. 2017. Personal identification based on content-independent EEG signal analysis. In Proceedings of the Chinese Conference on Biometric Recognition (CCBR'17). Springer, 537-544.

[87] S. H. Liew, Y. H. Choo, and Y. F. Low. 2013. Fuzzy-rough nearest neighbour classifier for person authentication using EEG signals. In Proceedings of the 2013 International Conference on Fuzzy Theory \& Its Applications (iFUZZY'13). 316321.

[88] F. Lin, K. W. Cho, C. Song, W. Xu, and Z. Jin. 2018. Brain password: A secure and truly cancelable brain biometrics for smart headwear. In Proceedings of the 16th International Conference on Mobile Systems, Applications, and Services (MobiSys'18). 296-309.

[89] Liu S. et al. 2014. Individual feature extraction and identification on EEG signals in relax and visual evoked tasks. In Biomedical Informatics and Technology (ACBIT'13), T. D. Pham, K. Ichikawa, M. Oyama-Higa, D. Coomans, and X. Jiang (Eds.). Communications in Computer and Information Science, Vol. 404. Springer, Berlin, Heidelberg. https://link.springer.com/chapter/10.1007/978-3-642-54121-6_29\#citeas.

[90] S. J. Luck. 2014. An Introduction to the Event-related Potential Technique. MIT press.

[91] L. Ma, J. W. Minett, T. Blu, and W. S.-Y. Wang. 2015. Resting state EEG-based biometrics for individual identification using convolutional neural networks. In Proceedings of the 37th Annual International Conference of the IEEE Engineering in Medicine \& Biology Society (EMBC'15). IEEE, 2848-2851.

[92] E. Maiorana and P. Campisi. 2018. Longitudinal evaluation of EEG-based biometric recognition. IEEE Transactions on Information and Forensic Security 13, 5 (2018), 1123-1138.

[93] E. Maiorana, G. E. Hine, and P. Campisi. 2015. Hill-climbing attacks on multibiometrics recognition systems. IEEE Transactions on Informationa and Forensic Security 10, 5 (2015), 900-915

[94] E. Maiorana, G. E. Hine, D. La Rocca, and P. Campisi. 2013. On the vulnerability of an EEG-based biometric system to hill-climbing attacks algorithms' comparison and possible countermeasures. In Proceedings of the IEEE 6th International Conference on Biometrics: Theory, Applications \& Systems (BTAS'13). 1-6.

[95] E. Maiorana, D. La Rocca, and P. Campisi. 2015. EEG-based biometric recognition using eigenbrains. In Proceedings of the 2015 IEEE International Conference on Multimedia \& Expo Workshops (ICMEW'15). IEEE, 1-6.

[96] E. Maiorana, D. La Rocca, and P. Campisi. 2016. Eigenbrains and eigentensorbrains: Parsimonious bases for EEG biometrics. Neurocomputing 171 (2016), 638-648.

[97] E. Maiorana, D. La Rocca, and P. Campisi. 2016. On the permanence of EEG signals for biometric recognition. IEEE Transactions on Information and Forensic Security 11, 1 (2016), 163-175.

[98] Z. Mao, W. X. Yao, and Y. Huang. 2017. EEG-based biometric identification with deep learning. In Proceedings of the 8th International IEEE/EMBS Conference on Neural Engineering (NER'17). IEEE, 609-612.

[99] E. Marasco and A. Ross. 2014. A survey on anti-spoofing schemes for fingerprint recognition systems. ACM Computing Surveys 47, 2 (Sept. 2014), 1-36.

[100] S. Marcel and J. R. Millán. 2007. Person authentication using brainwaves (EEG) and maximum a posteriori model adaptation. IEEE Transactions on Pattern Analysis and Machine Intelligence 29, 4 (Apr. 2007), 743-752.

[101] A. Mishra. 2010. Multimodal biometrics it is: Need for future systems. International fournal of Computer Applications 3, 4 (June 2010), 28-33.

[102] C. Miyamoto, S. Baba, and I. Nakanishi. 2009. Biometric person authentication using new spectral features of electroencephalogram (EEG). In Proceedings of the 2008 International Symposium on Intelligent Signal Processing \& Communication Systems (ISPACS'09). 1-4.

[103] M. Moreno-Revelo, M. Ortega-Adarme, D. H. Peluffo-Ordoñez, K. C. Alvarez-Uribe, and M. A. Becerra. 2017. Comparison among physiological signals for biometric identification. In Proceedings of the International Conference on Intelligent Data Engineering \& Automated Learning (IDEAL'17). Springer, 436-443.

[104] H. Morioka, A. Kanemura, J. Hirayama, M. Shikauchi, T. Ogawa, S. Ikeda, M. Kawanabe, and S. Ishii. 2015. Learning a common dictionary for subject-transfer decoding with resting calibration. NeuroImage 111 (2015), 167-178. 
[105] Z. Mu, J. Hu, and J. Min. 2016. EEG-based person authentication using a fuzzy entropy-related approach with two electrodes. Entropy 18, 12 (2016), 432.

[106] T. Nakamura, V. Goverdovsky, and D. P. Mandic. 2018. In-ear EEG biometrics for feasible and readily collectable real-world person authentication. IEEE Transactions on Information and Forensic Security 13, 3 (2018), 648-661.

[107] I. Nakanishi, S. Baba, and C. Miyamoto. 2009. EEG based biometric authentication using new spectral features. In Proceedings of the 2009 International Symposium on Intelligent Signal Processing \& Communication Systems (ISPACS'09). 651-654.

[108] I. Nakanishi, H. Fukuda, and S. Li. 2013. Biometric verification using brain waves toward on-demand user management systems: Performance differences between divided regions in $\alpha-\beta$ wave band. In Proceedings of the 6 th International Conference on Security of Information \& Networks (SIN'13). ACM, 131-135.

[109] I. Nakanishi and M. Hattori. 2017. Biometric potential of brain waves evoked by invisible visual stimulation. In Proceedings of the 2017 International Conference on Biometrics \& Kansei Engineering (ICBAKE'17). IEEE, 94-99.

[110] I. Nakanishi, K. Ozaki, and S. Li. 2012. Evaluation of the brain wave as biometrics in a simulated driving environment. In Proceedings of the 2012 International Conference on Biometrics Special Interest Group (BIOSIG'12). 1-5.

[111] B. Nguyen, D. Nguyen, W. Ma, and D. Tran. 2017. Investigating the possibility of applying EEG lossy compression to EEG-based user authentication. In Proceedings of the 2017 International foint Conference on Neural Networks (IFCNN'17). IEEE, 79-85.

[112] P. Nguyen, D. Tran, X. Huang, and W. Ma. 2013. Motor imagery EEG-based person verification. In Advanced Computer Intelligence, I. Rojas, G. Joya, and J. Cabestany (Eds.). Lecture Notes in Computer Science, Vol. 7903. Springer, Berlin, Heidelberg. https://link.springer.com/chapter/10.1007/978-3-642-38682-4_46\#citeas.

[113] P. Nguyen, D. Tran, X. Huang, and D. Sharma. 2012. A proposed feature extraction method for EEG-based person identification. In Proceedings of the International Conference on Artificial Intelligence (ICAI'12). 1-6.

[114] T. Nishimoto, Y. Azuma, H. Morioka, and S. Ishii. 2017. Individual identification by resting-state EEG using common dictionary learning. In Proceedings of the International Conference on Artifical Neural Networks (ICANN'17). Springer, 199-207.

[115] J. Onton and S. Makeig. 2006. Information-based modeling of event-related brain dynamics. Progress in Brain Research 159 (2006), 99-120.

[116] R. Palaniappan. 2004. Method of identifying individuals using VEP signals and neural network. IEE Proceedings: Scientific Measurement Technology 151, 1 (Jan. 2004), 16-20.

[117] R. Palaniappan. 2008. Two-stage biometric authentication method using thought activity brain waves. International fournal of Neural Systems 18, 1 (2008), 59-66.

[118] R. Palaniappan, S. Andrews, I. P. Sillitoe, T. Shira, and R. Paramesran. 2016. Improving the feature stability and classification performance of bimodal brain and heart biometrics. In Advanced Signal Processing and Intellingent Recognition Systems. Springer, 175-186.

[119] R. Palaniappan and D. P. Mandic. 2007. Biometrics from brain electrical activity: A machine learning approach. IEEE Transactions on Pattern Analysis and Machine Intelligence 29, 4 (Apr. 2007), 738-742.

[120] R. Palaniappan and D. P. Mandic. 2007. EEG based biometric framework for automatic identity verification. fournal of VLSI Signal Processing and System Signal, Image, and Video Technology 49, 2 (2007), 243-250.

[121] R. Palaniappan and K. V. R. Ravi. 2006. Improving visual evoked potential feature classification for person recognition using PCA and normalization. Pattern Recognition Letters 27, 7 (2006), 726-733.

[122] J. Pardey, S. Roberts, and L. Tarassenko. 1996. A review of parametric modelling techniques for EEG analysis. Medical Engineering and Physiology 18, 1 (1996), 2-11.

[123] V. Pasupathinathan. 2009. Hardware-based Identification and Authentication Systems. Ph.D. Dissertation. Macquarie University.

[124] V. Patel, M. Burns, R. Chandramouli, and R. Vinjamuri. 2017. Biometrics based on hand synergies and their neural representations. IEEE Access 5 (2017), 13422-13429.

[125] V. M. Patel, N. K. Ratha, and R. Chellappa. 2015. Cancelable biometrics: A review. IEEE Signal Processing Magazine 32, 5 (2015), 54-65.

[126] M. Pelillo. 2013. Similarity-Based Pattern Analysis and Recognition. Springer-Verlag London.

[127] T. Pham, W. Ma, D. Tran, P. Nguyen, and D. Phung. 2013. EEG-based user authentication in multilevel security systems. In Advanced Data Mining Applications, H. Motoda, Z. Wu, L. Cao, O. Zaiane, M. Yao, and W. Wang (Eds.). Lecture Notes in Computer Science, Vol. 8347. Springer, Berlin, Heidelberg. https://ink.springer.com/chapter/10. 1007/978-3-642-53917-6_46\#citeas.

[128] T. Pham, W. Ma, D. Tran, P. Nguyen, and D. Phung. 2014. Multi-factor EEG-based user authentication. In Proceedings of the 2014 International foint Conference on Neural Networks (IfCNN'14). IEEE, 4029-4034.

[129] T. Pham, W. Ma, D. Tran, D. S. Tran, and D. Phung. 2015. A study on the stability of EEG signals for user authentication. In Proceedings of the 7th International IEEE/EMBS Conference on Neural Engineering (NER'15). 122-125. 
[130] E. Piciucco, E. Maiorana, O. Falzon, K. P. Camilleri, and P. Campisi. 2017. Steady-state visual evoked potentials for EEG-based biometric identification. In Proceedings of the 2017 International Conference on Biometrics Special Interest Group (BIOSIG'17). 1-5.

[131] M. Poulos, M. Rangoussi, and N. Alexandris. 1999. Neural network based person identification using EEG features. In Proceedings of the 1999 IEEE International Conference on Acoustics, Speech, \& Signal Processing (ICASSP'99), Vol. 2. 1117-1120.

[132] M. Poulos, M. Rangoussi, N. Alexandris, and A. Evangelou. 2002. Person identification from the EEG using nonlinear signal classification. Methods Inf. Med. 41, 1 (2002), 64-75.

[133] M. Poulos, M. Rangoussi, V. Chrissikopoulos, and A. Evangelou. 1999. Person identification based on parametric processing of the EEG. In Proceedings of the 6th IEEE International Conference on Electronics, Circuits \& Systems (ICECS'99), Vol. 1. IEEE, 283-286.

[134] M. D. Pozo-Banos, J. B. Alonso, J. R. Ticay-Rivas, and C. M. Travieso. 2014. Electroencephalogram subject identification: A review. Expert Systems Applications 41, 15 (Nov. 2014), 6537-6554.

[135] M. W. Rahman and M. L. Gavrilova. 2017. Emerging EEG and kinect face fusion for biometric identification. In Proceedings of the 2017 IEEE Symposium on Serial Computing Intelligence (SSCI'17). IEEE, 1-8.

[136] K. V. R. Ravi and R. Palaniappan. 2005. Leave-one-out authentication of persons using $40 \mathrm{~Hz}$ EEG oscillations. In Proceedings of the 2005 International Conference on "Computer as a Tool” (EUROCON'05), Vol. 2. 1386-1389.

[137] J. D. M. Rennie, L. Shih, J. Teevan, and D. R. Karger. 2003. Tackling the poor assumptions of naive Bayes text classifiers. In Proceedings of the 20th International Conference on Machine Learning (ICML'03). 616-623.

[138] A. Riera, A. Soria-Frisch, M. Caparrini, C. Grau, and G. Ruffini. 2007. Unobtrusive biometric system based on electroencephalogram analysis. EURASIP fournal on Advanced Signal Processing 2008, 1 (2007), 1-8.

[139] I. Rizwan-i-Haque, M. F. Khan, M. Saleem, and N. I. Rao. 2012. Network weight adjustment in a fractional Fourier transform based multi-channel brain computer interface for person authentication. In Proceedings of the 11th International Conference on Information Science, Signal Processing \& Their Applications (ISSPA'12). 900-905.

[140] D. Rodrigues, G. F. A. Silva, J. P. Papa, A. N. Marana, and X.-S. Yang. 2016. EEG-based person identification through binary flower pollination algorithm. Expert Systems Application 62 (2016), 81-90.

[141] A. Ross and A. K. Jain. 2004. Multimodal biometrics: An overview. In Proceedings of the 12th European Signal Processing Conference. 1221-1224.

[142] M. V. Ruiz-Blondet, Z. Jin, and S. Laszlo. 2016. CEREBRE: A novel method for very high accuracy event-related potential biometric identification. IEEE Transactions on Information and Forensic Security 11, 7 (2016), 1618-1629.

[143] M. V. Ruiz-Blondet, Z. Jin, and S. Laszlo. 2017. Permanence of the CEREBRE brain biometric protocol. Pattern Recognition Letters 95 (2017), 37-43.

[144] M. V. Ruiz-Blondet, N. Khalifian, B. C. Armstrong, Z. Jin, K. J. Kurtz, and S. Laszlo. 2014. Brainprint: Identifying unique features of neural activity with machine learning. In Proceedings of the 36th Annual Conference of the Cognitive Science Society. 827-832.

[145] M. V. Ruiz-Blondet, N. Khalifian, K. J. Kurtz, S. Laszlo, and Z. Jin. 2014. Brainwaves as authentication method: Proving feasibility under two different approaches. In Proceedings of the 40th Annual Northeast Bioengineering Conference (NEBEC'14). IEEE, 1-2.

[146] M. V. Ruiz-Blondet, S. Laszlo, and Z. Jin. 2015. Assessment of permanence of non-volitional EEG brainwaves as a biometric. In Proceedings of the 2015 IEEE International Conference on Identity, Security \& Behavior Analysis (ISBA'15). IEEE, 1-6.

[147] G. Safont, A. Salazar, A. Soriano, and L. Vergara. 2012. Combination of multiple detectors for EEG based biometric identification/authentication. In Proceedings of the 2012 IEEE International Carnahan Conference on Security Technology (ICCST'12). 230-236.

[148] P. Sajda, A. Gerson, K. R. Müller, B. Blankertz, and L. Parra. 2003. A data analysis competition to evaluate machine learning algorithms for use in brain-computer interfaces. IEEE Transactions on Neural Systems Rehabilitation Engineering 11, 2 (June 2003), 184-185.

[149] S. K. Saripalle, A. McLaughlin, R. Krishna, A. Ross, and R. Derakhshani. 2015. Post-mortem iris biometric analysis in Sus scrofa domesticus. In Proceedings of the 2015 IEEE International Conference on Biometrics Theory, Applications \& Systems (BTAS'15). IEEE, 1-5.

[150] G. Schalk, D. J. McFarland, T. Hinterberger, N. Birbaumer, and J. R. Wolpaw. 2004. BCI2000: A general-purpose brain-computer interface (BCI) system. IEEE Transactions on Biomedical Engineering 51, 6 (June 2004), 1034-1043.

[151] A. Schlögl and C. Brunner. 2008. BioSig: A free and open source software library for BCI research. IEEE Computer 41, 10 (Oct. 2008), 44-50.

[152] T. Schons, G. J. P. Moreira, P. H. L. Silva, V. N. Coelho, and E. J. S. Luz. 2017. Convolutional network for EEG-based biometric. In Iberoamerican Congress on Pattern Recognition (CIARP'17). Springer, 601-608. 
[153] H. A. Shedeed. 2011. A new method for person identification in a biometric security system based on brain EEG signal processing. In Proceedings of the 2011 World Congress on Information \& Communication Technology (WICT'11). IEEE, 1205-1210.

[154] J. G. Snodgrass and M. Vanderwart. 1980. A standardized set of 260 pictures: Norms for name agreement, image agreement, familiarity, and visual complexity. Journal of Experimental Psychology: Human Learning and Memory 6 , 2 (1980), 174.

[155] A. Soria-Frisch, A. Riera, and S. Dunne. 2010. Fusion operators for multi-modal biometric authentication based on physiological signals. In Proceedings of the 2010 IEEE International Conference on Fuzzy Systems (FUZZ'10). IEEE, 1-7.

[156] D. Steyrl, R. Scherer, O. Förstner, and G. R. Müller-Putz. 2014. Motor imagery brain-computer interfaces: Random forests vs regularized LDA-non-linear beats linear. In Proceedings of the 6th International Brain-Computer Interface Conference. 241-244.

[157] F. Su, L. Xia, A. Cai, and J. Ma. 2010. A dual-biometric-modality identification system based on fingerprint and EEG. In Proceedings of the 4th IEEE International Conference on Biometrics: Theory Applications \& Systems (BTAS'10). IEEE, 1-6.

[158] F. Su, L. Xia, A. Cai, and J. Ma. 2010. Evaluation of recording factors in EEG-based personal identification: A vital step in real implementations. In Proceedings of the 2010 IEEE International Conference on Systems, Man, \& Cybernetics (SMC'10). IEEE, 3861-3866.

[159] F. Su, L. Xia, A. Cai, Y. Wu, and J. Ma. 2010. EEG-based personal identification: From proof-of-concept to a practical system. In Proceedings of the 20th International Conference on Pattern Recognition (ICPR'10). IEEE, 3728-3731.

[160] F. Su, H. Zhou, Z. Feng, and J. Ma. 2012. A biometric-based covert warning system using EEG. In Proceedings of the 5th IAPR International Conference on Biometrics (ICB'12). IEEE, 342-347.

[161] D. P. Subha, P. K. Joseph, U. R. Acharya, and C. M. Lim. 2010. EEG signal analysis: A survey. Fournal of Medical Systems 34, 2 (2010), 195-212.

[162] S. Sun. 2008. Multitask learning for EEG-based biometrics. In Proceedings of the 19th International Conference on Pattern Recognition (ICPR'08). 1-4.

[163] M. Tangermann, K. R. Müller, A. Aertsen, N. Birbaumer, C. Braun, C. Brunner, R. Leeb, C. Mehring, K. J. Miller, G. R. Müller-Putz, G. Nolte, G. Pfurtscheller, H. Preissl, G. Schalk, A. Schlögl, C. Vidaurre, S. Waldert, and B. Blankertz. 2012. Review of the BCI competition IV. Frontiers of Neuroscience 6, 55 (July 2012), 1-31.

[164] D. M. J. Tax and R. P. W. Duin. 2004. Support vector data description. Machine Learning 54, 1 (Jan. 2004), 45-66.

[165] M. Teplan. 2002. Fundamentals of EEG measurement. Measurement Science Review 2, 2 (2002), 1-11.

[166] K. P. Thomas and A. P. Vinod. 2016. Biometric identification of persons using sample entropy features of EEG during rest state. In Proceedings of the 2016 IEEE International Conference on Systems, Man, \& Cybernetics (SMC'16). IEEE, 003487-003492.

[167] K. P. Thomas and A. P. Vinod. 2016. Utilizing individual alpha frequency and delta band power in EEG based biometric recognition. In Proceedings of the 2016 IEEE International Conference on Systems, Man, \& Cybernetics (SMC'16). IEEE, 004787-004791.

[168] K. P. Thomas, A. P. Vinod, et al. 2017. EEG-based biometric authentication using self-referential visual stimuli. In Proceedings of the 2017 IEEE International Conference on Systems, Man, \& Cybernetics (SMC'17). IEEE, 3048-3053.

[169] M. Trokielewicz, A. Czajka, and P. Maciejewicz. 2016. Post-mortem human iris recognition. In Proceedings of the 2016 International Conference on Biometrics (ICB'16). 1-6.

[170] C. E. M. Van Beijsterveldt and D. I. Boomsma. 1994. Genetics of the human electroencephalogram (EEG) and eventrelated brain potentials (ERPs): A review. Human Genetics 94, 4 (1994), 319-330.

[171] C. Vidaurre, T. H. Sander, and A. Schlögl. 2011. BioSig: The free and open source software library for biomedical signal processing. Computer Intelligence and Neuroscience 2011 (2011), 1-12.

[172] M. Wang, H. A. Abbass, and J. Hu. 2016. Continuous authentication using EEG and face images for trusted autonomous systems. In Proceedings of the 14th Annual Conference on Privacy, Security \& Trust (PST'16). IEEE, 368-375.

[173] S. Wang, Y. C. Yu, and L. Gabel. 2014. An application of the P300 event-related potential as a biometric. In Proceedings of the 40th Annual Northeast Bioengineering Conference (NEBEC'14). IEEE, 1-2.

[174] Y. Wang and L. Najafizadeh. 2016. On the invariance of EEG-based signatures of individuality with application in biometric identification. In Proceedings of the 38th Annual International Conference of the IEEE Engineering in Medicine \& Biology Society (EMBC'16). IEEE, 4559-4562.

[175] E. F. M. Wijdicks. 1995. Determing brain death in adults. Neurology 45 (May 1995), 1003-1011.

[176] E. F. M. Wijdicks, P. N. Varelas, G. S. Gronseth, and D. M. Greer. 2010. Evidence-based guideline update: Determing brain death in adults: Report of the quality standards subcommittee of the American academy of neurology. Neurology 74 (June 2010), 1911-1918.

[177] Q. Wu, B. Yan, Y. Zeng, C. Zhang, and L. Tong. 2018. Anti-deception: Reliable EEG-based biometrics with real-time capability from the neural response of face rapid serial visual presentation. Biomedical Engineering Online 17, 1 (2018), 55 . 
[178] Q. Wu, Y. Zeng, C. Zhang, L. Tong, and B. Yan. 2018. An EEG-based person authentication system with open-set capability combining eye blinking signals. Sensors 18, 2 (2018), 335.

[179] S. Yang, F. Deravi, and S. Hoque. 2016. Task sensitivity in EEG biometric recognition. Pattern Analysis and Applications (2016), 1-13.

[180] X.-S. Yang. 2012. Flower pollination algorithm for global optimization. In Proceedings of the International Conference on Unconventional Computing \& Natural Computing (UCNC'12). Springer, 240-249.

[181] A. Yazdani, A. Roodaki, S. H. Rezatofighi, K. Misaghian, and S. K. Setarehdan. 2008. Fisher linear discriminant based person identification using visual evoked potentials. In Proceedings of the 9th International Conference on Signal Process. (ICOSP'08). 1677-1680.

[182] S. K. Yeom, H. I. Suk, and S. W. Lee. 2013. Person authentication from neural activity of face-specific visual selfrepresentation. Pattern Recognition 46, 4 (2013), 1159-1169.

[183] L. Yu and H. Liu. 2003. Efficiently handling feature redundancy in high-dimensional data. In Proceedings of the 9th ACM SIGKDD International Conference on Knowledge Discovery \& Data Mining (KDD’03). 685-690.

[184] L. Yu and H. Liu. 2003. Feature selection for high-dimensional data: A fast correlation-based filter solution. In Proceedings of the 20th International Conference on Machine Learning (ICML'03). 1-8.

[185] X. Zhang, L. Yao, K. Chen, X. Wang, Q. Sheng, and T. Gu. 2017. DeepKey: An EEG and gait based dual-authentication system. arXiv Preprint arXiv:1706.01606 (2017), 1-20.

[186] X. Zhang, L. Yao, S. S. Kanhere, Y. Liu, T. Gu, and K. Chen. 2017. MindID: Person identification from brain waves through attention-based recurrent neural network. arXiv Preprint arXiv:1711.06149 (2017), 1-20.

[187] X. L. Zhang, H. Begleiter, B. Porjesz, W. Wang, and A. Litke. 1995. Event related potentials during object recognition tasks. Brain Research Bulletin 38, 6 (1995), 531-538.

[188] A. Zúquete, B. Quintela, and J. P. Silva Cunha. 2010. Biometric authentication using brain responses to visual stimuli. In Proceedings of the 3rd International Conference on Bio-inspired Systems \& Signal Processing (BIOSIGNALS'10). 103112

Received July 2016; revised September 2017; accepted June 2018 